(C)2008 IEEE. Personal use of this material is permitted. However, permission to reprint/republish this material for advertising or promotional purposes or for creating new collective works for resale or redistribution to servers or lists, or to reuse any copyrighted component of this work in other works must be obtained from the IEEE. 


\title{
Search, Neutral Evolution and Mapping in Evolutionary Computing: A Case Study of Grammatical Evolution
}

\author{
Dominic Wilson, Member, IEEE, Devinder Kaur, Senior Member, IEEE
}

\begin{abstract}
We present a new perspective of search in Evolutionary Computing (EC) by using a novel model for the analysis and visualization of genotype to phenotype maps. The model groups genes into quotient sets and shows their adjacencies. A unique quality of the quotient model is that it details geometric qualities of maps that are not otherwise easy to observe. The model shows how random mutations on genes make non-random phenotype preferences, based on the structure of a map. The interaction between such mutation-based preferences with fitness preferences is important for explaining population movements on neutral landscapes. We show the widespread applicability of our approach by applying it to different representations, encodings and problems including Grammatical Evolution (GE), Cartesian Genetic Programming, Parity and Majority Coding, OneMax, Needle-in-Haystack, Deceptive Trap and Hierarchical if-and-only-if. We also use the approach to address conflicting results in the neutral evolution literature and to analyze concepts relevant to neutral evolution including robustness, evolvability, tunneling and the relation between genetic form and function.

We use the model to develop theoretical results on how mapping and neutral evolution affects search in GE. We study the two phases of mapping in GE; these being transcription (i.e. unique identification of genes with integers), and translation (i.e. many-to-one mapping of genotypes to phenotypes). It is shown that translation and transcription schemes belong to equivalence classes, therefore the properties we derive for specific schemes are applicable to classes of schemes. We present a new perspective on population diversity. We specify conditions under which increasing degeneracy (by increasing codon size) or rearranging the rules of a grammar do not affect performance. It is shown that there is a barrier to nontrivial neutral evolution with the use of the natural transcription with modulo translation combination; a necessary but not sufficient condition for such evolution is at least three bits should change on mutation within a single codon. This barrier can be avoided by using Gray transcription. We empirically validate some findings.
\end{abstract}

Index Terms-Genotype to Phenotype map, Degeneracy, Redundancy, Grammatical Evolution, Search Distribution, Neutral Evolution.

Devinder Kaur is with the Electrical Engineering and Computer Science Department, University of Toledo, Toledo, Ohio, 43606 USA (Phone: 419530-8163; fax: 419-530-8146; e-mail: dkaur@ eecs.utoledo.edu).

Dominic Wilson is with the Electrical Engineering and Computer Science Department, University of Toledo, Toledo, Ohio, 43606 USA (e-mail: dowilson@eng.utoledo.edu).

\section{INTRODUCTION}

In many Evolutionary Computing (EC) approaches, a genotype-to-phenotype map is used to convert populations of genomes that inhabit a search space into expressions that can be evaluated to give fitness values. The mapping processes used usually have some implicit structure to them. By this we mean it is usually possible to prescribe the map as an algorithm, or to describe the map by use of a concise description, or a meaningful name. In contrast a randomly generated map usually requires a listing of genotypes and the associated phenotypes they represent. The structure of a map can be examined both from the perspective of how it defines genetic neighborhoods and phenotypic selectivity of genes. Symmetries inherent in a map can sometimes make it possible to develop a simplified model of the evolutionary process. Grammatical Evolution (GE) [1], [2], [3], [4], [5], [6], [7], [8], [9], [10], [11], [12], [13], [14] is an example of an Evolutionary Computing approach that uses prescriptive (algorithmic) maps to define a phenotype from a genome.

GE is a Genetic Programming system that uses a Genetic Algorithm to search the space of structures specified by some context free or attribute grammar. The grammar specifies ordered sets of derivation alternatives as the right hand terms of productions. This grammar restricts phenotypes to syntactically correct forms, avoiding problems with closure, typing being determined through its use. GE separates the search (genotype) space from the solution (phenotype) space, with the search space being composed of binary strings (genomes). The solution space is composed of expressions or programs based on the grammar and derived in general through many-to-one mappings of the genome.

Using a many-to-one (i.e. degenerate) map means that more than one genotype will be expressed as the same phenotype. This feature is usually justified based on the neutral theory of evolution [15]. The neutral theory of evolution hypothesizes that most genetic changes are neutral (i.e. they do not affect the fitness of the genome); it also states that most evolutionary changes are the result of random genetic drift among networks of neutral genomes. A neutral mutation is a mutation event that has no effect on the assessed fitness of a genome [6]. Ryan and O'Neill [5], [6] have studied the usefulness of 
neutral evolution in GE. These studies show that having a degenerate mapping process, (i.e. many codons selecting the same right term) can increase genetic diversity and performance in some applications. Looking more broadly at the current body of Evolutionary Computing literature, degenerate maps and neutrality have received a lot of attention and there is a mixed picture on the issue of their usefulness e.g. [16], [17], [18].

Neutral evolution is closely linked with the mapping process in use, so understanding neutral evolution requires some understanding of the stages of mapping. In GE, genotype-to-phenotype mapping involves a two stage process of transcription and translation. Transcription is the identification of segments of genome (known as genes or codons) with integer values. Translation is the use of the resulting integer value to make a derivation, based on a grammar. GE's two stage mapping approach will allow us to independently deal with genetic representation and phenotype surjection (i.e. many-to-one mapping). We will analyze GE transcription and translation models in this paper especially as they pertain to neutral evolution.

The main aim of this paper is to investigate how neutrality and mapping affect search in GE in particular and EC in general. Our approach is to:

- Simplify the analysis of search by grouping together and characterizing genetic material that show identical response on being affected by some search algorithm.

- Derive and empirically validate some general principles governing search in GE in the presence of neutrality as embodied by some choice of a mapping scheme.

Most of the literature on GE is based on the use of natural transcription (i.e. natural binary encoding) and modulo translation. The analyses in this paper will reflect this bias. However, in order to make our results more general, we will also introduce an alternative Gray transcription method.

The rest of this paper is organized as follows: Section II provides details on how GE works. The grammar used for the Santa-Fe problem [6] in the literature is introduced and used to explain how genomes are converted to executable code. Section III introduces notations and some definitions useful for subsequent sections. In section IV the concept of neutrality in the context of the codon is formalized and classes of neutrality for the codons are defined. Quotient sets are introduced and sample quotient graphs of neutral sets are shown. In section $\mathrm{V}$ we present theorems that relate the transcription and translation functions with neutrality. In section VI we extend the concept of neutrality from the single codon (gene) to the entire genome (individual). We then derive necessary conditions for neutral evolution of the genome. Section VII analyzes symmetries in right hand term orderings; through these symmetries we identify other translation schemes with identical search characteristics with the modulo scheme. In section VIII an alternative model using
Gray transcription is introduced and compared to the standard method of using natural transcription. In section IX experiments are done to exemplify and corroborate our theoretical findings. Section X discusses and analyzes related work on neutral evolution in EC and GE as well as related models in EC. Conclusions are drawn in the final section. An appendix contains quotient graphs of a variety of problems and encodings that have been used in the literature.

\section{How GRAmMATICAL EvOlUTION WORKS}

O'Neill and Ryan [7] provide a detailed explanation of how GE works. The language to be generated by GE is specified using a Backus Naur Form (BNF) grammar. Fig. 1 shows an example grammar consisting of a series of production rules mapping a set of non-terminal symbols to a set of terminals that are defined in the language. A special non-terminal is the start symbol.

Fig. 1 shows the grammar used for the Santa-Fe Ant Trial problem [6], [7], [70]. The Santa-Fe Ant Trial problem is a standard test problem in both GP and GE that involves the automatic programming of an artificial ant to find 89 pieces of food located in a discontinuous trail within a specified number of time steps. In the grammar, the symbol "::=" denotes that the non-terminal on the left of the production rule can be derived into the symbol that appears on the right in one step. The pipe symbol I is used to separate alternative derivations. The numbers in parenthesis to the right are there to ease reference to particular right hand terms in the discussion. Production (a) of Fig. 1 shows that definitions can be recursive.

A linear genome, made up of a variable number of fixed length genes, (or codons) is used to control how the BNF grammar definition is mapped to an actual program. In most implementations the codon is an 8 bit binary string, however other bit sizes and non-binary encoding types are possible.

The codons are transcribed to their corresponding integer values. This transformation is usually the identification of the codon with its decimal value. The transcribed values are then used to decide which derivation to carry out whenever there is a choice between two or more alternatives. A derivation is selected by using the following algorithm (which is referred to as modulo translation) [2], [5], [6]:

\section{(Integer Codon Value) Mod (Number of derivation alternatives for current non-terminal)}

Consider for example the production (e) of Fig. 1, given the non-terminal " $<\mathrm{Op}>$ " there are three alternative derivations to select from. If we assume a codon string of "00011101", the integer corresponding to the codon being read is 29 , then 29 MOD $3=2$ resulting in the selection of "move()" as the terminal. The system walks through the genome by reading a new codon each time a production with two or more possible derivations has to be used. 


\begin{tabular}{|c|c|c|c|}
\hline \multicolumn{4}{|c|}{$\begin{array}{l}\text { Non-terminals }=\{\text { Code, Line, Expr, If-statement, Op }\} \\
\text { Terminals }=\{\text { left }(), \operatorname{right}(), \text { move }(), \text { food_ahead }(), \text { else, } \\
\text { if, }\{,\},(,), ;\}\end{array}$} \\
\hline \multicolumn{4}{|c|}{ Start symbol $::=<$ Code $>$} \\
\hline $\begin{array}{l}\mathrm{Th} \\
\text { (a) }\end{array}$ & $\begin{array}{l}\text { of production } \\
<\text { Code }>:=\end{array}$ & $\begin{array}{l}\text { es is: } \\
\quad<\text { Line }> \\
\mid<\text { Code }><\text { Line }>\end{array}$ & $\begin{array}{l}\text { (0) } \\
(1)\end{array}$ \\
\hline $\begin{array}{l}\text { (b) } \\
\text { (c) }\end{array}$ & $\begin{array}{l}<\text { Line }>::= \\
<\text { Expr }>:=\end{array}$ & $\begin{array}{l}<\text { Expr }> \\
<\text { If-statement }> \\
\mid<\text { Op }>\end{array}$ & $\begin{array}{l}\text { (0) } \\
(1)\end{array}$ \\
\hline (d) & $<$ If-statemen & 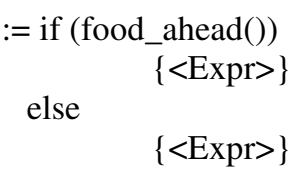 & \\
\hline (e) & $<\mathrm{Op}\rangle \quad::=$ & $\begin{array}{l}\operatorname{left}() \\
\mid \operatorname{right}() \\
\operatorname{lmove}()\end{array}$ & $\begin{array}{l}(0) \\
(1) \\
(2)\end{array}$ \\
\hline
\end{tabular}

Fig. 1. BNF grammar for the Santa-Fe Ant Trial problem.

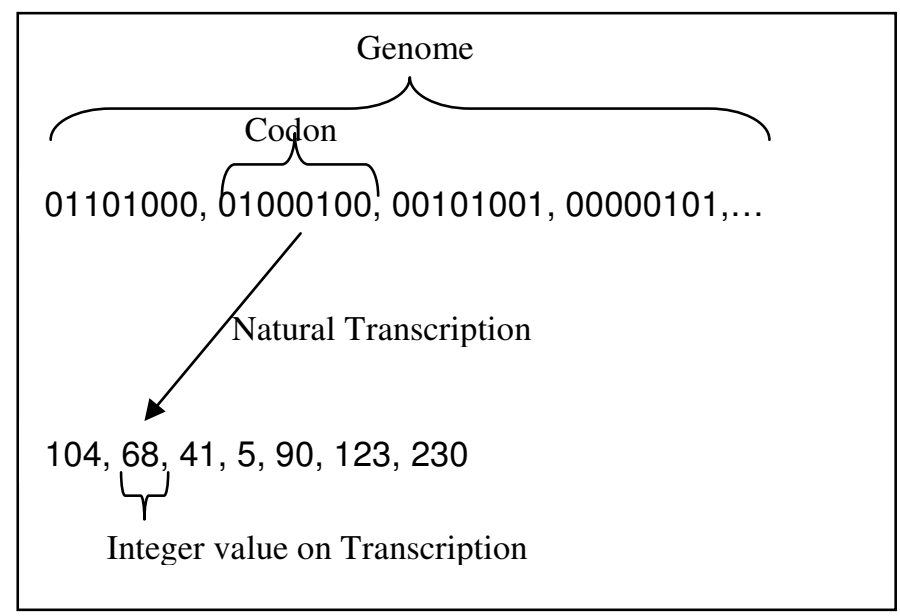

Fig. 2. Example codon sequence

Fig. 2 shows the binary and corresponding integer sequence of a genome made up of 7 codons. For the binary string only the first four codon details are shown. The integer sequence is the result of the transcription process. We now show how this integer sequence is mapped to executable code. The translation part of the mapping process starts with the start symbol $<$ Code $>$; because there are more than one derivation possible for this nonterminal (i.e. 2), we use the stated formula to get 104 MOD $2=0$, and choose the derivation labeled (0). $<$ Code $>$ is replaced with $<$ Line $>$. The next step involves the replacement of the leftmost non-terminal " $<$ Line $>$ by " $<$ Expr $>$ ". Because there is only one derivation possible for "<Line>", a new codon value is not used. The next step involves calculating $68 \mathrm{MOD} 2=0$, that specifies the use of the derivation:
$<$ Expr $>\quad::=<$ If-statement $>$.

Full resolution of the codon sequence yields the program:

$$
\begin{aligned}
& \text { if (food_ahead()) } \\
& \text { else } \\
& \text { \{move();\} } \\
& \{\text { left();\} }
\end{aligned}
$$

Fig. 3. The fully derived program based on grammar of Fig. 1 and codon sequence of Fig. 2

The mapping system has transformed a binary string into a program. Sometimes the length of the string is not sufficient to completely map a genome into a program, in those cases the codon sequence can be reused repeatedly in a process known as wrapping. An arbitrary upper limit is usually chosen to limit the number of wrappings as explained in [19]. Those programs not fully resolved within the limit are given a minimum fitness and subsequently removed from the population, due to their low selection probability.

A Genetic Algorithm involving the use of selection, mutation and recombination is used to evolve the fittest genome for creating the program. The evolutionary aspect of $\mathrm{GE}$ is language independent. Apart from such practical considerations as setting a maximum genome size, in theory the types of functions that can be generated are only limited by what the grammar in use can express.

\section{NOTATIONS AND SOME DEFINITIONS}

Context-free grammars (CFG) have long been used for programming language implementation, description, and analysis [20]. Our formal definition of CFG's is based on that of McGettrick [21]. A CFG is a 4-tuple $C F G=\{N, T, P, S\}$ where

- $N$ is a finite set of non-terminals;

- $T$ is a finite set of terminals disjoint with $N$;

- $P$ is a finite set of productions or rewrite rules, $P: \alpha \rightarrow \beta$, with $\alpha \in N, \beta \in N \cup T$.

- $S \in N$ is a special nonterminal called the start symbol.

If a production $\alpha \rightarrow \beta$ acts on a string $\rho \alpha \kappa$ we obtain the immediate derivation $\rho \alpha \kappa \rightarrow \rho \beta \kappa$ by replacing $\alpha$ with $\beta$. We will simply refer to an immediate derivation as a derivation; if a derivation is not immediate it will be further qualified.

It is usual for a set of productions:

$\alpha \rightarrow \beta_{0} ; \alpha \rightarrow \beta_{1} ; \cdots ; \alpha \rightarrow \beta_{i} ; \cdots ; \alpha \rightarrow \beta_{n}$,

that have the same left part to be abbreviated as:

$\alpha \rightarrow \beta_{0}\left|\beta_{1}\right| \cdots\left|\beta_{i}\right| \cdots \mid \beta_{n}$;

the set of right parts $\left\{\beta_{0}, \beta_{1}, \cdots, \beta_{i}, \cdots, \beta_{n}\right\}$ being the derivation alternatives of $\alpha$. We will refer to this abbreviated 
form that involves multiple right parts as a production rule.

A GE grammar system is a 5-tuple $G=\{N, T, P, S, \Omega\}$, consisting of the context free grammar $\{N, T, P, S\}$ and a set of all $\mathrm{m}$-bit binary strings $\Omega=\left\{x: x=\sum_{2}^{m}\right\}$ called codons. The cardinality of $\Omega, \operatorname{card}(\Omega)$ is $2^{m}$. The codons select a derivation when a non-terminal has multiple alternative derivations. If the $i^{\text {th }}$ production rule has $n_{i}$ alternative derivations, a production rule can be further specified as:

$$
\alpha_{i} \stackrel{\Psi\left(n_{i}, x\right)}{\longrightarrow} \beta_{i, 0}\left|\beta_{i, 1}\right| \cdots \mid \beta_{i, n_{i}-1},
$$

with $\Psi\left(n_{i}, x\right)=\operatorname{Tla}\left(n_{i}, \operatorname{Tcr}(x)\right)$ being a mapping function composed of a transcription function $\operatorname{Tcr}: x \rightarrow \mathbb{Z}$ and a translation function Tla: $(\mathbb{Z} \times \mathbb{Z}) \rightarrow \mathbb{Z} ; \mathbb{Z}$ being the set of nonnegative integers. Note that $\Psi$ is independent of any problemspecific parameters.

Transcription in GE is the method of uniquely identifying the binary codon string with an integer label. We will also use the notations $x$, and $\Omega=\left\{x: 0 \leq x \leq 2^{m}-1\right\}$ to refer to the integer label of a codon on transcription and the set of such integer labels. Also the notation $x_{i}$ will refer to the state of the binary string at position $i$ (i.e. the allele), $x_{i} \in\{0,1\}$.

In the natural transcription model each codon's state has a one-to-one correspondence with an integer value:

$$
x=\sum_{j=0}^{m-1} x_{j} 2^{j} .
$$

For modulo translation a production rule is selected by the map:

$$
\Psi\left(n_{i}, x\right)=x \bmod n_{i}
$$

\section{A. Degeneracy, Redundancy and Neutrality}

Due to the many different ways that the words Degeneracy, Redundancy and Neutrality have been used in the EC literature it is prudent to identify how they will be used in this paper.

A map is degenerate when multiple codon values map to the same right hand term, the multiplicity determines the degree of degeneracy of the map. We will not use degeneracy as a synonym for neutrality. Neutrality is associated with some characteristics of the behavior of a degenerate map. Various types of neutrality are defined and discussed in detail in section IV.

Some of the EC literature (especially those related to genetic algorithms), use redundancy as a synonym for degeneracy (e.g. [22], [23]). Radcliffe [24], however introduced a different notion of redundancy and distinguished between degenerate representations (where more than one genotype encodes the same phenotype), and redundant representations (where part of the genotype is not used in the construction of the phenotype). Within Genetic Programming we observe the similar concepts of functional redundancies [25], [26] (i.e. functions that are semantically identical but syntactically different), and redundancies due to introns (i.e. gene not used in the mapping process). We classify these as semantic forms of degeneracy. Semantic degeneracy is intrinsic to the problem being solved and is usually not under the control of the EC practitioner. In contrast syntactic forms of degeneracy are generated by a deliberate use of a systematically constructed degenerate map. The mapping function of associating genotype to phenotype can be understood without requiring the evaluation of a phenotype. Examples of such maps include the use of a larger than necessary codon size in GE, and parity, truth table and majority coding as used in Galvan and Poli [18].

Rothlauf and Goldberg [81] defined a representation as being uniformly redundant if all phenotypes are represented by the same number of different genotypes. In GE, all phenotypes are represented by the same number of different genotypes only when the number of derivation alternatives is a power of two. For the rest of this work we limit the use of the term redundancy to refer to functional redundancy and uniform redundancy (i.e. when all phenotypes are represented by the same number of different genotypes).

\section{B. Phenotype}

In EC, the term "phenotype" is usually reserved for a final representation of a genome along the process of transformation pursuant to being assigned fitness. We relax this definition to include intermediate representations of a genome along this process. This means a Genotype to Phenotype map can be a map to an intermediate form. The particular (intermediate or final) form being referenced will be clear from the context of use.

The usefulness of this approach is that it allows us to analyze encodings (e.g. parity coding) and representations (e.g. natural transcription used with modulo translation) without reference to a specific problem. Using the same framework, we will also be able to analyze specific problems (e.g. OneMax) and problems using a particular encoding or representation (e.g. OneMax using parity coding).

Specific to GE, when we refer to the phenotypic (or derivation) value of a codon we mean the particular choice made by the codon (i.e. the index of the right hand term the codon selects during mapping). When we refer to the phenotypic value of a genome we mean the complete set of choices made by the codons in that genome in order to generate the complete phenotype.

\section{Derivation Context}

We will refer to the production rule in which the codon will be expressed as the context of use of the codon. If two production rules have the same count of derivation alternatives, a codon will choose derivations with the same index in either context. As we will be doing analysis independent of semantics, we will find it convenient to refer to the context simply by the count of derivation alternatives. For example if we refer to a codon in a context " 6 ", we mean the attributes of that codon when it is being used to express a production with six derivation alternatives. 


\section{NEUTRALITY AND THE CODON}

In this section we look at neutrality from the perspective of a codon. Our approach can be summarized as collecting sets of codons that have the same phenotype and search behavior and use the characteristics and dynamics of those sets to understand neutral evolution.

Consider a genome in which only a single codon's state is allowed to change (by random mutation). The phenotypic search distribution associated with that codon is the probability distribution of phenotype values obtained on varying that codon's state. If the mapping is degenerate (such that more than one state of the codon selects the same phenotype), we can talk of a neutral network existing between the codon states that select the same phenotype. The approach of using search distributions to characterize neutrality is originally due to Toussaint [57]. A trivial neutral network is one in which neutrality has no effect on search, because the neutral members project the same search distribution. Consequently the expected progress of evolution of any codon is the same as that for any other codon it is trivially neutral with. In contrast a nontrivial neutral network is one in which although all neutral members have the same fitness, the search distributions they project are not the same. In nontrivial neutral networks some codon states can select the same phenotype without having the same variation ability (ability to generate more fit variants) and robustness (ability to resist harmful phenotypic change on mutation) as others. Due to differences in search distributions, these neutral states can have different effective fitness $[27,28,29]$ as measured by the number of offspring they produce, due to their assessed fitness and the effect of genetic operators on them.

We continue this section with a simple example that will help to show the reasoning behind our approach to neutrality. Using the example, we explain derivation neutrality, which is neutrality related to codons being indistinguishable on applying the selection part of the evolutionary process. A matrix representation for mapping is then developed and its connection to derivation neutrality is clarified. We then explain search neutrality, which is neutrality related to codons being indistinguishable on applying the mutation part of the evolutionary process. Using our example, we show how the neighborhoods of codons influence search and how some codons give identical search distributions on mutation.

\section{A. Derivation and Search Neutrality}

Example 1: Consider a simple GE system having only one production rule with 3 derivation alternatives as shown in Fig. 4. Assume a genome consists of a single codon, the codon size being 3 bits. The right-hand terms 50,10 and 20, are the phenotype values of the codon associated with the first, second and third derivation choices respectively. Natural transcription and modulo translation are being used. Table 1 shows the mappings of all the 3 -bit codons of example 1 . The string 010 , for instance, transcribes to the integer 2, and since $2 \bmod 3=2$, the string selects the third right hand term which is 20 (using zero-based indexing).

\begin{tabular}{|ll|}
\hline$<$ value $>::=$ & 50 \\
& $\mid 10$ \\
& $\mid 20$ \\
\hline
\end{tabular}

Fig. 4. Example production rule with 3 derivation alternatives.

Table 1. Mapping 3-bit codons to derivation choice for example 1.

\begin{tabular}{ccc}
\hline $\begin{array}{l}\text { Codon } \\
\text { string }\end{array}$ & $\begin{array}{l}\text { Integer } \\
\text { value on } \\
\text { natural } \\
\text { transcription }\end{array}$ & $\begin{array}{l}\text { Derivation } \\
\text { choice on } \\
\text { modulo } \\
\text { translation }\end{array}$ \\
\hline 000 & 0 & 50 \\
001 & 1 & 10 \\
010 & 2 & 20 \\
011 & 3 & 50 \\
100 & 4 & 10 \\
101 & 5 & 20 \\
110 & 6 & 50 \\
111 & 7 & 10 \\
\hline
\end{tabular}

Using the example, for the set of strings $\Omega_{0}=\{000,011,110\}$ we map to the same phenotypic value 50; likewise for the set of strings $\Omega_{1}=\{001,100,111\}$ and $\Omega_{2}=\{010,101\}$ we map to the values 10 and 20 respectively. We refer to sets $\Omega_{0}, \Omega_{1}$ and $\Omega_{2}$ as derivation neutral sets as each set selects the same derivation or right hand term.

Definition: A derivation neutral set of codons is a set that maps to the same derivation (i.e. right hand term) in a context specified by the nonterminal being replaced.

Because the strings in a derivation neutral set select the same right hand term, they give the same phenotype; there is no way to differentiate between members of such a derivation neutral set based on their phenotype.

We can identify codons by their unique transcription values, as such we can say $\Omega_{0}=\{0,3,6\}, \Omega_{1}=\{1,4,7\}$ and $\Omega_{2}=\{2,5\}$. Also we can identify derivation neutral sets by the zero-based index that identifies the right-hand choice they make, giving our derivation neutral sets as $[0]:=\{0,3,6\},[1]:=\{1,4,7\}$ and $[2]:=\{2,5\}$. This naming scheme coincides with the congruence of the integer values modulo the number of rules.

Assuming m-bit coding, all translation schemes (including modulo translation) map $2^{m}$ integer choices unto $n_{i}$ derivation choices, with $n_{i} \leq 2^{m}$. We can represent the said integer values and their derivation choices as unitary vectors $X$ and $\Psi$ of sizes $2^{m}$ and $n_{i}$ respectively, with: 


$$
X_{i}=\left\{\begin{array}{ll}
1, & i=x \\
0 & \text { otherwise }
\end{array} .\right.
$$

For instance codons 000 and 101 have integer values 0 and 5 and are represented as vectors

$$
\begin{aligned}
& X=\left\{\begin{array}{llllllll}
1 & 0 & 0 & 0 & 0 & 0 & 0 & 0
\end{array}\right\}^{T} \text { and } \\
& X=\left\{\begin{array}{lllllllll}
0 & 0 & 0 & 0 & 0 & 1 & 0 & 0
\end{array}\right\}^{T} \text { respectively. The vector }
\end{aligned}
$$
representation of $\Psi$ is:

$$
\Psi_{i}=\left\{\begin{array}{l}
1, \quad i=\text { index of derivation choice } \\
0 \text { otherwise }
\end{array} .\right.
$$

The derivation choices choosing the first, second and third choice are therefore respectively: $\Psi=\left\{\begin{array}{lll}1 & 0 & 0\end{array}\right\}^{T}, \quad\left\{\begin{array}{lll}0 & 1 & 0\end{array}\right\}^{T}$, and $\left\{\begin{array}{lll}0 & 0 & 1\end{array}\right\}^{T}$. Note that these representations form bases (i.e. they are linearly independent and span their respective vector spaces).

With these representations, we can construct a $n_{i} \times 2^{m}$ linear operator, $A$, that maps codons to derivation choices, with $\Psi=A X$. The definition of such a matrix is:

$$
A_{i, j}=\left\{\begin{array}{l}
1, \text { if integer } j \text { maps to choice } i \\
0 \text { otherwise }
\end{array} .\right.
$$

For our example,

$$
\begin{aligned}
& A=\left[\begin{array}{lllll}
A_{0,0} & A_{0,1} & . & . & A_{0,7} \\
A_{1,0} & A_{1,1} & . & . & A_{1,7} \\
A_{2,0} & A_{2,1} & \cdot & . & A_{2,7}
\end{array}\right], \\
& =\left[\begin{array}{llllllll}
1 & 0 & 0 & 1 & 0 & 0 & 1 & 0 \\
0 & 1 & 0 & 0 & 1 & 0 & 0 & 1 \\
0 & 0 & 1 & 0 & 0 & 1 & 0 & 0
\end{array}\right] .
\end{aligned}
$$

For every row of $A$, the set of indexes of columns that have a unit value is a derivation neutral set. We will find this matrix representation of mapping useful in explaining search neutrality, which we now discuss.

Fig. 5 represents the codons of our example as vertices on a cube. This representation shows the neighborhood structure between strings. It can be seen that each string in the derivation neutral set [0] (shown with white vertices) has as neighbors two strings in set [1] (Gray vertices) and one string in set [2] (that with the brick pattern); We can therefore infer that for all strings belonging to set [0], mutation of a single bit chosen at random leads to the same phenotypic search distribution (i.e. same probability that the resulting mutant string belongs to sets [1] or [2]). It is not difficult to see that even for the six possible 2-bit mutations and the one possible 3-bit mutation the search distributions are identical for all members of [0]. We therefore denote [0] as a set composed of search neutral members.
Definition: A search neutral set of codons is a set of derivation neutral codons that has the same phenotypic search distribution on mutation.

By our definition search neutral sets are (proper or improper) subsets of derivation neutral sets. The value of this characterization is that when we know a codon belongs to a search neutral set, say [0], then we have all the information necessary to understand its phenotype and search behavior. We can replace the codon with some other randomly chosen member of the same search neutral set and expect identical search behavior. Set [2] is an example where the derivation neutral members are not search neutral. On single bit mutation, for instance, codon 010 has strings in [0] as neighbors, whereas codon 101 has neighbors in [1]. When this happens we divide the set into search neutral sets, labeling them with both the derivation name and some additional lettering, and list the content of each individual set. We thus get $[2 \mathrm{a}]=\{2\}$ and $[2 \mathrm{~b}]=\{5\}$.

Another way of looking at search neutrality is to consider each bit of an m-bit binary codon string being mutated with some fixed probability $\mu$. The search distribution induced by mutation on the codon can be modeled by applying an $2^{m} \times 2^{m}$ mutation rate matrix $M$ on the codon with $M_{i, j}=\mu^{d(x, y)}(1-\mu)^{m-d(x, y)}$, where $d(x, y)$ is the Hamming distance between the present codon $x$, and its potential mutated value $y$.

The mutation rate matrix for our example 3-bit system is:

$$
\begin{aligned}
& 000 \\
& 001 \\
& 010
\end{aligned}\left[\begin{array}{cccccc}
000 & 001 & 010 & . & \cdot & 111 \\
(1-\mu)^{3} & \mu(1-\mu)^{2} & \mu(1-\mu)^{2} & . & . & \mu^{3} \\
\mu(1-\mu)^{2} & (1-\mu)^{3} & \mu^{2}(1-\mu) & & & \\
\mu(1-\mu)^{2} & \mu^{2}(1-\mu) & (1-\mu)^{3} & & & \\
& & & & & \\
\cdot & & & \cdot & & \\
\cdot & & & & \cdot & \\
\cdot & & & & \cdot & \\
\mu^{3} & & & & & (1-\mu)^{3}
\end{array}\right]
$$

The genotypic search distribution on mutation of a codon is $M X$, where $X$ is the unitary representation of the codon. Its phenotypic search distribution $\Phi_{X}$ is given by:

$$
\Phi_{X}=A M X \text {. }
$$

Let $\Phi=A M$, for the example, its transpose is:

$$
\begin{aligned}
& 0\left[(1-\mu)^{3}+2 \mu^{2}(1-\mu) \quad \mu^{3}+2 \mu(1-\mu)^{2} \quad \mu(1-\mu)^{2}+\mu^{2}(1-\mu)\right] \\
& 1 \quad \mu^{3}+2 \mu(1-\mu)^{2} \quad(1-\mu)^{3}+2 \mu^{2}(1-\mu) \quad \mu(1-\mu)^{2}+\mu^{2}(1-\mu) \\
& \begin{array}{l|lll}
2 & 3 \mu(1-\mu)^{2} & 3 \mu^{2}(1-\mu) & \mu^{3}+(1-\mu)^{3}
\end{array} \\
& \Phi^{T}=3(1-\mu)^{3}+2 \mu^{2}(1-\mu) \quad \mu^{3}+2 \mu(1-\mu)^{2} \quad \mu(1-\mu)^{2}+\mu^{2}(1-\mu) \\
& { }_{4} \quad \mu^{3}+2 \mu(1-\mu)^{2} \quad(1-\mu)^{3}+2 \mu^{2}(1-\mu) \quad \mu(1-\mu)^{2}+\mu^{2}(1-\mu) \\
& 5 \quad 3 \mu^{2}(1-\mu) \quad 3 \mu(1-\mu)^{2} \quad \mu^{3}+(1-\mu)^{3} \\
& 6(1-\mu)^{3}+2 \mu^{2}(1-\mu) \quad \mu^{3}+2 \mu(1-\mu)^{2} \quad \mu(1-\mu)^{2}+\mu^{2}(1-\mu) \\
& 7\left[\begin{array}{ccc}
\mu^{3}+2 \mu(1-\mu)^{2} & (1-\mu)^{3}+2 \mu^{2}(1-\mu) & \mu(1-\mu)^{2}+\mu^{2}(1-\mu)
\end{array}\right]
\end{aligned}
$$

We see, from its transpose, that the phenotypic search distribution for columns 0,3 and 6 of $\Phi$ are identical and equal to: 


$$
\left[\begin{array}{c}
(1-\mu)^{3}+2 \mu^{2}(1-\mu) \\
\mu^{3}+2 \mu(1-\mu)^{2} \\
\mu(1-\mu)^{2}+\mu^{2}(1-\mu)
\end{array}\right]
$$

This concurs with our visual analysis of search neutrality. Likewise we can observe the identical search distributions for columns 1, 4 and 7 associated with set [1], and the different search distributions of columns 2 and 5 meaning [2a] and [2b] have different search distributions.

\section{B. $\quad$ Search Neutral Sets As Quotient Sets And Graphs}

Let $\pi: \Omega \rightarrow\left\{\Omega_{i}\right\}$ represent the partitioning of codons into pairwise disjoint and nonempty search neutral sets $\Omega_{i}$, with $\cup \Omega_{i}=\Omega$. Because members of each set $\Omega_{i}$ have the same search distribution, the partitioning $\pi$, is such that for any two partitions $\Omega_{i}, \Omega_{j}$, the number of neighbors of any codon $x \in \Omega_{i}$ to $\Omega_{j}$ is independent of the exact value of the codon and depends only on the partition indexes $i$ and $j$. The search neutral members $x \in \Omega_{i}$ are an equivalence class. Any member can be chosen as a class representative.

Such equitable partitioning is a well known concept in graph theory and system aggregation theory [30], [31], [32], [33]. Exact partitioning and aggregation have also been analyzed in Evolutionary Computing (e.g. Vose [34], Rowe et al. [35]). These partitioning have been based on genetic features without reference to the phenotype. See section X.C for a related discussion on EC models.

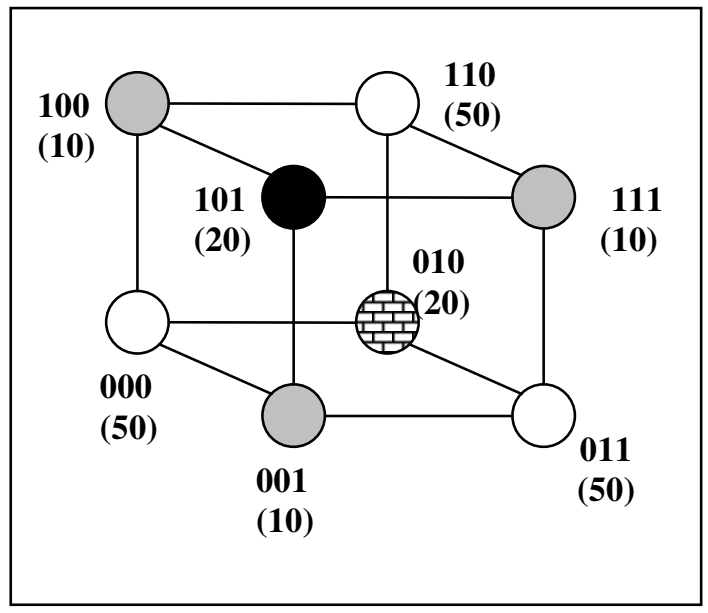

Fig. 5. codons (and their derivation choices) represented as vertices on a cube

In general there is more than one way of achieving such partitioning at different levels of fineness (or coarseness). A partitioning $\pi: \Omega \rightarrow\left\{\Omega_{i}\right\}_{1 \leq i \leq n}$ is finer than another partitioning $\pi^{\prime}: \Omega \rightarrow\left\{\Omega_{i}^{\prime}\right\}_{1 \leq i \leq n^{\prime}}$ if $\pi \neq \pi^{\prime}$ and every set of $\Omega_{j}^{\prime}$ is a union of sets of $\Omega_{i}$. $\pi^{\prime}$ is said to be coarser than $\pi$. What we are interested in is obtaining the coarsest search neutral sets. The finest partitioning is achieved by having every partition contain a single codon only. The coarsest partitioning can be found by grouping together the search neutral subsets of derivation neutral sets.

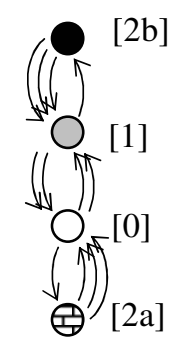

Fig. 6. Quotient adjacency graph for 3-bit codon and 3 derivation choices using natural transcription and modulo translation.

The procedure for obtaining search neutral sets is easily automated by partitioning derivation neutral sets into sets with all members having the same phenotypic search distribution. The use of such sets is not limited to GE and can be used in other areas of Evolutionary Computing. The appendix shows them applied to a variety of encodings and problems not related to GE.

A quotient set of search neutral codons is a set having each search neutral set represented by a single member. We can represent quotient set assignment of codons by a matrix $\mathrm{Q}$ where:

$Q_{i, j}=\left\{\begin{array}{l}1, \text { if integer } j \text { maps to search neutral set } i \\ 0 \text { otherwise }\end{array}\right.$.

For our example:

$$
Q=\underset{[1]}{[0]} \underset{[2 a]}{[2 b]}\left[\begin{array}{cccccccc}
0 & 1 & 2 & 3 & 4 & 5 & 6 & 7 \\
1 & 0 & 0 & 1 & 0 & 0 & 1 & 0 \\
0 & 1 & 0 & 0 & 1 & 0 & 0 & 1 \\
0 & 0 & 1 & 0 & 0 & 0 & 0 & 0 \\
0 & 0 & 0 & 0 & 0 & 1 & 0 & 0
\end{array}\right]
$$

The adjacencies between search neutral sets can be displayed with the aid of quotient sets. To obtain the adjacencies we can take any member of each search neutral set and find their search distribution for single bit mutations. Alternatively let $\bar{H}$ and $H$ represent the quotient adjacency matrix, and the adjacency matrix of the hypercube respectively. If $\bar{H}$ contains all adjacencies specified by $H$, then assigning any codon $X$ to its search neutral set, $Q X$, and then applying the quotient adjacency matrix should be equivalent to finding its adjacency on the hypercube, $H X$, and then assigning the result to search neutral sets, i.e.

$\bar{H} Q X=Q H X$,

Which yields:

$\bar{H}=Q H Q^{T}\left(Q Q^{T}\right)^{-1}$.

The product $Q Q^{T}$ is a square diagonal matrix with diagonal elements equal to the number of codons within the relevant 
search neutral set. Thus $Q Q^{T}$ is full rank and invertible and $\bar{H}$ always exists. The following matrix represents the adjacencies between search neutral sets for our 3-bit example:

$$
\bar{H}=\underset{[0]}{[1]}\left[\begin{array}{cccc}
{[0]} & {[1]} & {[2 a]} & {[2 b]} \\
{[2 a]} & 2 & 1 & 0 \\
2 & 0 & 0 & 1 \\
3 & 0 & 0 & 0 \\
0 & 3 & 0 & 0
\end{array}\right] .
$$

The elements of the adjacency matrix representing the number of directed adjacent transitions between and within search neutral sets. For instance any member of search neutral set [0] has no adjacent neighbor in search neutral set [0], 2 adjacent neighbors in set [1], one neighbor in set [2a] and no adjacent neighbor in set [2b]. The adjacency matrix can be plotted as a quotient adjacency graph (which is a directed multigraph with each search neutral set represented as a vertex). We will refer to the quotient adjacency graph simply as a quotient graph. The quotient graph represents an entire search neutral set as a single representative entity and shows its relation with other search neutral sets; this way the effects of mutation and the interaction of search neutral sets become easier to understand. Fig. 6 is the quotient graph for our example. The shading if the nodes in Fig. 6 correspond to that of the nodes on the cube in Fig. 5.

Fig. 6 shows some of the advantages of the quotient representation. One advantage is that we are able to represent the adjacencies of an 8-node cube by a reduced model (a 4node quotient model for our example). Another advantage is we can visually assess the phenotypic neighborhood structure. For our example we see that a transition between the only two nontrivially neutral sets i.e. sets [2a] and [2b] requires at least 3 bit changes as their representative nodes are distance 3 apart. We can also see which transitions do and don't affect search. There are no arcs that start and end on the same node in Fig. 6 so we can deduce that every single bit mutation results in a mutant with a different search distribution from its parent.

Fig. 7 shows quotient graphs using codon sizes of 3 and 4 bits for derivation choices of 2 to 7 . Table 2 shows the number of search neutral sets for codon sizes between 2 and 10 bits and derivation choices between 2 and 24. Patterns and symmetries apparent in the graphs and table suggest certain properties which will be examined in the next section. These include the observation that arcs that start and end on the same node are only present when the number of derivation alternatives is a power of 2 . Such arcs indicate mutations that do not change the phenotype (derivation choice) or the search distribution. Also with derivation alternatives being a power of 2, note that all derivation neutral sets are search neutral. A distance of at least 3 between derivation neutral choices that are not search neutral can be observed (for instance, in the case of our example between [2a] and [2b]). As Table 2 shows the number of search neutral sets needed to model a codon, it highlights the possible reduction in model size on using search neutral sets. Without the use of search neutral sets, an m-bit codon requires a $2^{m} \times 2^{m}$ matrix to model its behavior at the genetic level.

\section{Quotient Mutation Rate Matrix}

We can define a rate matrix based on the quotient representation that represents the dynamics of mutation at the quotient level. Let $\bar{M}$ represent the quotient rate matrix. For this matrix to exactly model mutation at the quotient level, assigning any codon $X$ to its search neutral set, $Q X$, and then applying the quotient rate matrix should be equivalent to carrying out the normal course of mutation, $M X$, and then assigning the result to search neutral sets, i.e.

$\bar{M} Q X=Q M X$,

this yields:

$\bar{M}=Q M Q^{T}\left(Q Q^{T}\right)^{-1}$

We can show that $\bar{M}$ always exists by using similar arguments to that used for showing that the quotient adjacency matrix always exists.

The quotient rate matrix $\bar{M}$ can be written as:

$$
\bar{M}=\bar{M}_{\text {trivial }}+\bar{M}_{\text {nontrivial,neutral }}+\bar{M}_{\text {non-neutral }}
$$

$\bar{M}_{\text {trivial }}$ is composed of all diagonal elements of $\bar{M}$. $\bar{M}_{\text {trivial }}$ represents the cases of no mutation and trivial mutation. Any changes to the diagonal elements will have no effect on search. The off-diagonal elements of $\bar{M}$ represent nontrivial search. $\bar{M}_{\text {nontrivil, neutral }}$ is composed of the elements of $\bar{M}$ related to derivation neutral, but not search neutral search. $\bar{M}_{\text {non-neutral }}$ is composed of the elements of $\bar{M}$ related to search that is not derivation neutral.

For our example the quotient mutation rate matrix is:

$$
\begin{aligned}
& \text { [0] } \\
& \text { [1] } \\
& \text { [2a] }
\end{aligned}
$$

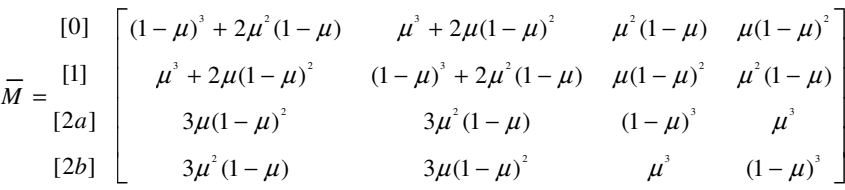

\section{D. $\quad$ Correlated Mutational Drives}

An important fact that quotient graphs reveal is that there is sometimes a preferred directions of evolutionary movement due to random mutations. This preference is independent of fitness. We will refer to it as a mutational drive (as opposed to the usual fitness drive). The arcs in Fig. 6, for example, show that there is a net mutational drive from both edge nodes towards the central nodes.

Correlated mutational drives are present because the arrangement of phenotypes correlates with distances between genotypes. Such correlated mutational drives are sometimes the unsuspecting result of the use of highly structured mapping schemes that cause correlations of the effects of random mutations. 
Table 2. Number of search neutral sets for different number of derivation alternatives and codon sizes, using natural transcription and modulo translation.

\begin{tabular}{|c|c|c|c|c|c|c|c|c|c|}
\hline \multirow{2}{*}{ 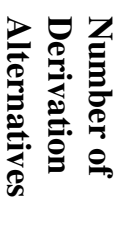 } & \multicolumn{9}{|c|}{ Codon Size in bits } \\
\hline & 2 & 3 & 4 & 5 & 6 & 7 & 8 & 9 & 10 \\
\hline 2 & 2 & 2 & 2 & 2 & 2 & 2 & 2 & 2 & 2 \\
\hline 3 & 3 & 4 & 5 & 6 & 7 & 8 & 9 & 10 & 11 \\
\hline 4 & 4 & 4 & 4 & 4 & 4 & 4 & 4 & 4 & 4 \\
\hline 5 & & 6 & 9 & 12 & 16 & 20 & 25 & 30 & 36 \\
\hline 6 & & 6 & 8 & 10 & 12 & 14 & 16 & 18 & 20 \\
\hline 7 & & 8 & 12 & 18 & 27 & 36 & 48 & 64 & 80 \\
\hline 8 & & 8 & 8 & 8 & 8 & 8 & 8 & 8 & 8 \\
\hline 9 & & & 12 & 18 & 27 & 36 & 48 & 64 & 80 \\
\hline 10 & & & 12 & 18 & 24 & 32 & 40 & 50 & 60 \\
\hline 11 & & & 16 & 32 & 48 & 72 & 108 & 162 & 243 \\
\hline 12 & & & 12 & 16 & 20 & 24 & 28 & 32 & 36 \\
\hline 13 & & & 16 & 32 & 64 & 96 & 144 & 216 & 324 \\
\hline 14 & & & 16 & 24 & 36 & 54 & 72 & 96 & 128 \\
\hline 15 & & & 16 & 24 & 36 & 54 & 81 & 108 & 144 \\
\hline 16 & & & 16 & 16 & 16 & 16 & 16 & 16 & 16 \\
\hline 17 & & & & 24 & 36 & 54 & 81 & 108 & 144 \\
\hline 18 & & & & 24 & 36 & 54 & 72 & 96 & 128 \\
\hline 19 & & & & 32 & 64 & 128 & 256 & 512 & 768 \\
\hline 20 & & & & 24 & 36 & 48 & 64 & 80 & 100 \\
\hline 21 & & & & 32 & 64 & 96 & 144 & 216 & 324 \\
\hline 22 & & & & 32 & 64 & 96 & 144 & 216 & 324 \\
\hline 23 & & & & 32 & 64 & 128 & 256 & 512 & 1024 \\
\hline 24 & & & & 24 & 32 & 40 & 48 & 56 & 64 \\
\hline
\end{tabular}

\section{NeUTRALITY AND MAPPING IN GE}

We present some theorems that relate the transcription and translation functions with neutrality. These theorems are based on observations of the quotient graphs in Fig. 7. The first theorem shows that if there is uniform redundancy, when using natural transcription and modulo translation, degeneracy does not affect the search process.

Theorem 1: Using natural transcription and modulo translation, all derivation neutral sets are search neutral when $n_{i}=2^{k}, 0 \leq k \leq m$.

Proof: Because $[x]:=x \bmod 2^{k}=\sum_{i=0}^{k-1} x_{i} 2^{i}$, for all members of $[x]$, any mutation on bits within $k \leq i \leq m$ are irrelevant to derivation or search. Any mutation on bits within $0 \leq i \leq k-1$ leads to identical search.

Theorem 1 shows that for uniform redundancy (when $n_{i}=2^{k}$ ), any degeneracy affects neither search nor derivation.

The following two lemmas provide a lower limit on the number of bit changes required for nontrivial neutral mutations. We note that a nontrivially neutral mutation is a mutation event in which the mutant has the same derivation value as the original codon but has a different search distribution. The lemmas show that using natural transcription and modulo translation:

- When the number of derivation alternatives is not a power of 2, a single bit change cannot be derivation neutral;

- Any 2-bit mutation that is derivation neutral is also search neutral, and therefore trivially neutral.

For the case of the number of derivation alternatives being a power of 2, Theorem 1 has shown that all neutrality is trivial. Therefore the following two lemmas, when combined with Theorem 1, show that there can be no mutation event that is nontrivially neutral that involves a change of less than 3 bits.

Lemma 1 : Using natural transcription and modulo translation, for $n_{i} \neq 2^{k}$, a single bit mutation cannot be derivation neutral.

Proof: On single bit mutation of an m-bit codon, the difference between the new and old codon integer value is $x^{\prime}-x= \pm 2^{i}, 0 \leq i \leq m-1$. If mutation is neutral then $x^{\prime}-x=k n_{i}$, where $\mathrm{k}$ is an integer.

$\therefore k n_{i}= \pm 2^{i}$. This is impossible for $n_{i} \neq 2^{k}$.

From Lemma 1, with $n_{i} \neq 2^{k}$, summing the rates for $1 \mathrm{bit}$ changes, we expect a codon to change its derivation choice at a rate above $m \mu(1-\mu)^{m-1}$; this is a large proportion of mutated codons. For $\mu=0.01$ and $m=8$ for instance, more than $96 \%$ of mutated codons will change their derivation choice. The fact that there are no two neighboring codons with the same derivation choice shows a low level of robustness.

Lemma 2: Using modulo translation, any 2-bit mutation that is derivation neutral is also search neutral.

Proof: Without loss of generality assume an m-bit codon with the bits to be mutated being $x_{i}$ and $x_{j}, i \neq j$. The mutant codon then has (m-2) bits identical with the unmutated codon and $x_{i}^{\prime}=\bar{x}_{i}$ and $x_{j}^{\prime}=\bar{x}_{j}$, where $x_{i}^{\prime}$ refers to the $i^{\text {th }}$ bit of the mutant. To establish search neutrality we go through the search distributions for the codon and its mutant and show that they are the same.

The Hamming distances of the codon and its mutant to the $2^{m-1}$ codons with values $\bar{x}_{i}, x_{j}$ or $x_{i}, \bar{x}_{j}$ at positions $i$ and $j$, are identical, therefore there is the same search distribution to this set of codons from either the unmutated codon or the mutant. 


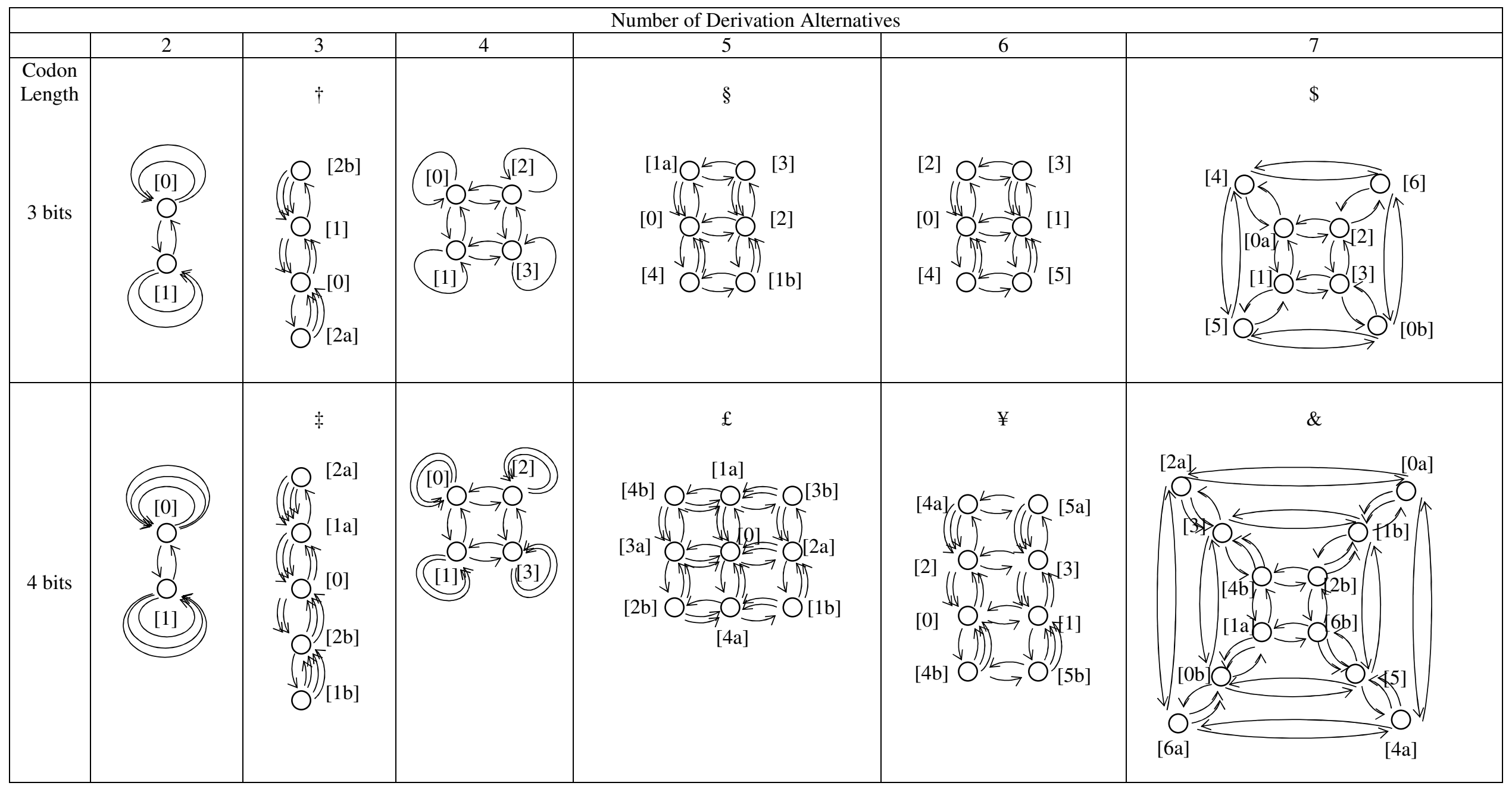

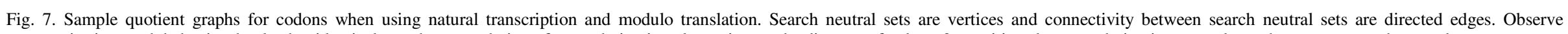
symmetries in search behavior that lead to identical search on reordering of some derivation alternatives and a distance of at least 3 transitions between derivation neutral sets that are not search neutral.

$\dagger[2 \mathrm{a}]=\{2\},[2 \mathrm{~b}]=\{5\} . \ddagger[1 \mathrm{a}]=\{1,4,7,13\},[1 \mathrm{~b}]=\{10\},[2 \mathrm{a}]=\{2,8,11,14\},[2 \mathrm{~b}]=\{5\}$.

$\S[1 \mathrm{a}]=\{1\},[1 \mathrm{~b}]=\{6\} . £[1 \mathrm{a}]=\{1,11\},[1 \mathrm{~b}]=\{6\},[2 \mathrm{a}]=\{2,7\},[2 \mathrm{~b}]=\{12\},[3 \mathrm{a}]=\{8,13\},[3 \mathrm{~b}]=\{3\},[4 \mathrm{a}]=\{4,14\},[4 \mathrm{~b}]=\{9\}$.

$¥[4 \mathrm{a}]=\{10\},[4 \mathrm{~b}]=\{4\},[5 \mathrm{a}]=\{11\},[5 \mathrm{~b}]=\{5\}$.

$\$[0 \mathrm{a}]=\{0\},[0 \mathrm{~b}]=\{7\} . \&[0 \mathrm{a}]=\{0\},[0 \mathrm{~b}]=\{7,14\},[1 \mathrm{a}]=\{15\},[1 \mathrm{~b}]=\{1,8\},[2 \mathrm{a}]=\{2\},[2 \mathrm{~b}]=\{9\},[4 \mathrm{a}]=\{4\},[4 \mathrm{~b}]=\{11\},[6 \mathrm{a}]=\{6\},[6 \mathrm{~b}]=\{13\}$ 
For the remaining $2^{m-1}$ codons, consider a codon $y$, with values $x_{i}, x_{j}$ at positions $i$ and $j$, this codon will be at a Hamming distance of 2 less to the unmutated codon that to the mutant. Its counterpart (which is identical but with $\bar{x}_{i}, \bar{x}_{j}$ at positions $i$ and $j$ ) will be at a Hamming distance of 2 more to the unmutated codon than to the mutant. Furthermore both the codon $y$ and its counterpart are derivation neutral based on:

$x \bmod n=x^{\prime} \bmod n \Rightarrow(x+c) \bmod n=\left(x^{\prime}+c\right) \bmod n$,

where $y=x+c$. As such we have equal numbers of derivation neutral codons at equal distances from the codon and its mutant meaning the codon and its mutant project the same search distribution.

Corollary 1: Using modulo translation, there must be at least 3-bit changes for nontrivial neutral evolution.

Proof: This follows from Theorem 1, Lemma 1 and Lemma 2.

We now look at the concept of equivalent transcription models. The importance of this concept is that it will allow us to determine the size and structure of classes of transcription models that will give identical behaviors. An example of the usefulness of this is that we will be able to derive different transcription models that give identical behavior to natural transcription. We show that subgroups of transcription models are equivalent in terms of having identical genetic neighborhood structures; We give the cardinality of this subgroup and show the number of transcription model that are not equivalent for a given codon size. Furthermore we prove Theorem 2 (which states that search neutrality is preserved by equivalent transcription models) showing that equivalent transcription models give identical search behavior and performance.

Equivalent structures based on the neighborhoods of binary strings have been studied by Rowe et al. [36]. There are $2^{m}$ !different ways in which the state of an m-bit codon can be uniquely labeled using integer labels from the set $\Omega=\left\{x: 0 \leq x \leq 2^{m}-1\right\}$. Any two such labeling (alternatively we can refer to them as representations, encodings [36] or transcription models) $r, s$ are equivalent if they maintain the same neighborhood structure, i.e.

$$
\forall x, y \in \Omega: d_{r}(x, y)=1 \Leftrightarrow d_{s}(x, y)=1,
$$

where $d_{r}(x, y)$ is the Hamming distance between codons with labels $x$ and $y$ in representation $r$. A representation can be converted to any other representation by some permutation. A permutation that transforms one representation into an equivalent representation is known as an automorphism. As such an automorphism by definition is a subgroup of the group of permutations that preserves the adjacency structure between labels. It has been proven by Harary [38] that any automorphism of such representations can be described as the action of a unique combination of an exclusive-or mask and a permutation on the bits of the strings. Automorphisms form a group under function composition [36,37], this means that application of different automorphisms will always preserve the adjacency structure of the original representation.

Table 3. Example of equivalent representations.

\begin{tabular}{ccc}
\hline Codon & Representation & Representation \\
string & $A$ & $B$ \\
\hline 00 & 0 & 1 \\
01 & 1 & 3 \\
10 & 2 & 0 \\
11 & 3 & 2 \\
\hline
\end{tabular}

Table 3 shows an example of equivalent representations of a 2-bit codon. Representation $A$ is the usual decimal representation of a binary string. In both representations $A$ and B the label " 2 ", for instance, is one bit different from the labels " 0 " and " 3 ". As such the Hamming distance is preserved between representations. The automorphism that transforms representation A to B is composed of applying the exclusive-or mask "10" to representation A and then exchanging the position of the two resulting bits.

For an $m$-bit codon, there are $2^{m}$ ! possible transcription models. There are also $2^{m}$ possible exclusive-or masks and $m$ ! permutations; As such there are $2^{m} m$ ! unique automorphisms. The number of distinct transcription models that have different neighborhood structures is therefore $2^{m} ! / 2^{m} m !$ or equivalently $\left(2^{m}-1\right) ! / m$ !

Theorem 2: Search neutrality is preserved by equivalent transcription models.

Proof: The mutation operator $M_{x, y}=\mu^{d(x, y)}(1-\mu)^{m-d(x, y)}$ is based on the Hamming distances between vertices, therefore if a bijection maintains such distances search neutrality is preserved. Equivalent representations can be decomposed into bit permutations and exclusive-or mask operations. For bit permutation it is obvious that Hamming distances are maintained. For masks, consider a mask $\chi$ operating on vertices $x$ and $y$. The Hamming distance after application of the mask is:

$$
\begin{aligned}
& d((\chi \oplus x),(\chi \oplus y)) \\
& =\chi \oplus x \oplus \chi \oplus y=x \oplus y .
\end{aligned}
$$

Since The Hamming distance after application of the mask is equal to that before the application, the Hamming distance is preserved.

Corollary 2: Permuting and bit-masking codon bit positions as part of the transcription process does not affect search.

Proof: This is a direct consequence of Theorem 2.

What this corollary shows is that every transcription scheme is a member of a subgroup of transcription schemes that are 
equivalent in behavior. Assuming a codon size of $m$ bits, there are $2^{m}$ possible masks and $m$ ! permutations; therefore the number of members of the subgroup is $2^{m} m$ !. Obtaining an equivalent transcription scheme simply involves bit-masking and/or permuting the bits of a transcription scheme. We can therefore see that there is a set of transcription schemes that will give identical search as the natural transcription scheme. This includes all transcription models of the form:

$$
x=\sum_{j=0}^{m-1}\left(\chi_{j} \oplus x_{\sigma(j)}\right) 2^{j} \text {. }
$$

Where $\chi$ is an m-bit mask, $\oplus$ is the bitwise exclusive-or operator, and $x_{\sigma(j)}$ is the $j^{\text {th }}$ bit of $x$ after permutation. Table 3 for instance shows two of the eight possible transcription models that have identical behavior as the natural transcription model on a 2-bit codon.

\section{NEUTRALITY AND THE GENOME}

In this section we examine the neutrality of genomes. We have looked at search distributions projected by a codon when that codon is expressed in a single context. For genomes made up of many codons and used with grammars having many rules, we need to analyze search distributions in all possible contexts specified by the grammar in use.

Although a codon will retain its value on transcription, it can select different right terms depending on the context in which it is being expressed; this change of derivation choice on reinterpretation of a codon in a new context is also known as intrinsic polymorphism. A codon context change can happen due to some crossover event or a mutation event on a preceding codon.

Recall that a search neutral set of codons is a set of derivation neutral codons that has the same phenotypic search distribution on mutation. In a situation with multiple productions defining many contexts in which a codon can be expressed we extend this to the following:

Definition: A search neutral set of codons is a set of codons that are derivation neutral in all contexts and have the same phenotypic search distribution on mutation in all contexts.

We also define search neutrality for the genome as:

Definition: Two or more genomes are search neutral if they are of the same length, and pair-wise their codons are search neutral.

Proposition 1: Using modulo translation, there must be at least 3-bit changes within a single codon for nontrivial neutral evolution of a genome.

Proof: Using Theorem 1, Lemma 1 and Lemma 2 a single or double mutation within any single codon, in any context, is either trivially neutral or not derivation neutral.

Table 4: 3-bit codons and their associated integer values on Gray transcription

\begin{tabular}{ccc}
\hline $\begin{array}{c}\text { Gray coded } \\
\text { Codon }\end{array}$ & $\begin{array}{c}\text { Codon binary } \\
\text { value }\end{array}$ & $\begin{array}{c}\text { Integer value on } \\
\text { transcription }\end{array}$ \\
\hline 000 & 000 & 0 \\
\hline
\end{tabular}

\begin{tabular}{lll}
\hline 001 & 001 & 1 \\
011 & 010 & 2 \\
010 & 011 & 3 \\
110 & 100 & 4 \\
111 & 101 & 5 \\
101 & 110 & 6 \\
100 & 111 & 7 \\
\hline
\end{tabular}

From observing the quotient graphs for 6 derivation alternatives in Fig. 7, we note that all codon members of any search neutral set for 6 derivation alternatives are also search neutral when those codons are used in a situation with either 2 or 3 derivation alternatives. This is because 2 and 3 are factors of 6 . We will first prove that this is a general result involving factors and use the result to provide a sufficient condition for genomes to be search neutral.

Lemma 3: Let $k$ and $l$ be two positive integers. Every search neutral set in a context with $k * l$ derivation alternatives is a subset of a search neutral set in a context with $k$ derivation alternatives.

Proof: The search distribution for a context with $k * l$ derivation alternatives is given by

$\Phi_{k * l}=A_{k * l} M$,

where $A_{k * l}$ is the map associating genes with the $k * l$ derivation alternatives, $M$ is the $2^{m} \times 2^{m}$ mutation matrix for codon size $m$.

The search distribution for a context with $k$ derivation alternatives is given by:

$$
\Phi_{k}=A_{k} M \text {. }
$$

where $A_{k}$ is the map associating genes with the $k$ derivation alternatives. Because $k$ is a factor of $k * l$,

$A_{k}=\left[\mathrm{I}_{k} \mathrm{I}_{k} \cdots \mathrm{I}_{k}(l \text { times })\right]^{*} A_{k * l}$,

where $\mathrm{I}_{k}$ is the identity matrix of size $k$.

$\therefore \Phi_{k}=\left[\mathrm{I}_{k} \mathrm{I}_{k} \cdots \mathrm{I}_{k}(l \text { times })\right]^{*} \Phi_{k * l}$

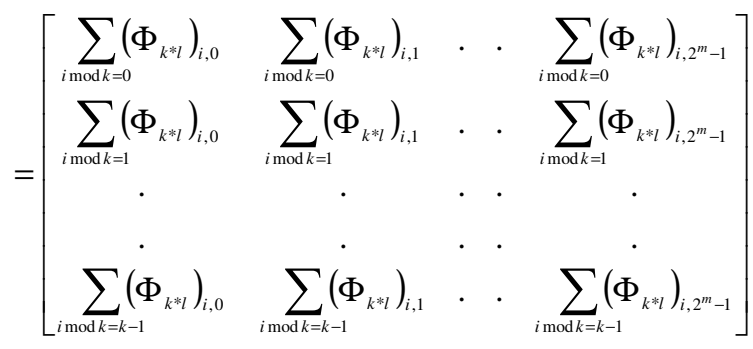

Because $\Phi_{k}$ is the combination of rows of $\Phi_{k^{* l}}$ any search neutral pair of codons in $n_{k^{* l}}$ is also search neutral in $n_{k}$

Corollary 3: If the counts of the production alternatives of a grammar with $r$ productions are $\left(n_{0}, n_{1}, \ldots, n_{r}\right)$ in a GE system using m-bit codons, natural transcription and modulo translation, two or more genomes are search neutral if pair- 
wise their codons are search neutral modulo $\operatorname{LCM}\left(n_{0}, n_{1}, \ldots, n_{r}\right)$, with $\operatorname{LCM}\left(n_{0}, n_{1}, \ldots, n_{r}\right) \leq 2^{m}$ and the genomes are of the same length.

Proof: Let $n=\operatorname{LCM}\left(n_{0}, n_{1}, \ldots, n_{r}\right)$. Lemma 3 shows that any codons that are search neutral in a context specified by $n$ are search neutral in a context specified by all of the factors of n.

Search neutral genomes derive the same tree (are derivation neutral), and are virtually identical as seen by the GE system even in the presences of wrapping and crossover schemes which do not depend on actual codon values. Treating them as identical gives a better idea of effective diversity present in a population than using measures based on the states of the individual bits of genomes. It is clear for instance that for a system with $\operatorname{LCM}\left(n_{0}, n_{1}, \ldots, n_{r}\right)=2^{k}, \quad 0<k \leq m$, while measures of diversity based on allele states will increase with codon sizes greater than $k$, measures based on our definition of search neutrality will not change, signaling that the extra alleles do not affect performance.

Another implication of Lemma 3 is that for $\operatorname{LCM}\left(n_{0}, n_{1}, \ldots, n_{r}\right) \leq 2^{m}$, if we know the derivation and search behavior of a codon in a context $n=\operatorname{LCM}\left(n_{0}, n_{1}, \ldots, n_{r}\right)$, then we can tell its derivation and search behavior in every context. In short the context $n$ has all the information necessary for exact modeling of any codon.

\section{SYMMETRIES IN RIGHT TERM ORDERING}

From the symmetries in the quotient graphs it can be determined that certain permutations of derivation alternatives yield identical search distributions while others do not. The importance of this fact is that it allows us to identify what ordering of derivation alternatives will give the same search distribution (hence performance) as other orderings, and which will give different distributions.

Consider a case where $n_{i}=2^{k}$ (i.e. $n_{i}$ is a power of 2) with natural transcription and modulo translation. The quotient graph is a k-cube with unique vertex labels from the set of derivation alternatives $\left\{\beta_{i, 0}\left|\beta_{i, 1}\right| \ldots \mid \beta_{i, n_{i}-1}\right\}$. There are $n_{i}$ ! possible orderings of the derivation alternatives. As established by Harary [38], any automorphism of a hypercube can be described as a unique combination of a mask and a permutation. Because there are $2^{k}$ possible masks and $k$ ! permutations, then there are $2^{k} k$ ! unique automorphisms. The number of distinct representations of the set of derivation alternatives is therefore $n_{i} ! / 2^{k} k$ ! or equivalently $\left(n_{i}-1\right) ! / k$ ! For example a production with 4 derivation alternatives $\{a|b| c \mid d\}$, has the following three distinct representations: $\{a|b| c \mid d\}$, $\{a|b| d \mid c\}$ and $\{a|c| d \mid b\}$; the set of 24 possible orderings of the derivation alternatives can be divided into 3 disjoint subsets, each subset being equivalent to one of the representations given above.

There are also some symmetries for $n_{i} \neq 2^{k}$. For instance for the 4 bit codon with $n_{i}=3$, any permutation of positions 1 and 2 (leaving position 0 unchanged) i.e. $\{a|b| c\} \Leftrightarrow\{a|c| b\}$ is an automorphism. This means the number of distinct representations from permuting the three derivation alternatives is $3 ! / 2=3$.

In general the automorphisms can be identified by the set of permutation matrices $\left\{P: P R P^{-1}=R\right\}$ where $R$ is the adjacency matrix of the quotient graph.



Fig. 8 Sample quotient graphs for codons when using Gray transcription and modulo translation. Search neutral sets are vertices and connectivity between search neutral sets are directed edges.

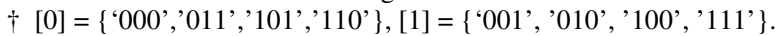

$\ddagger[0]=\left\{{ }^{0} 0000\right.$ ', '0011', '0101', '0110', '1001', '1010', '1100', '1111' $\},[1]=$ \{'0001', '0010', '0100', '0111', '1000', '1011', '1101','1110'\}.

$£[0]=\left\{{ }^{\prime} 000,, 110^{\prime}\right\},[1]=\left\{{ }^{\prime} 0011^{\prime},,^{\prime} 111^{\prime}\right\},[2]=\left\{{ }^{\prime} 011^{\prime},{ }^{\prime} 101 '\right\},[3]=$ $\left\{{ }^{\prime} 010,, ' 100\right.$ ' $\}$.

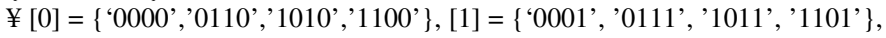

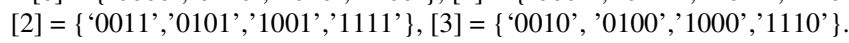

\section{GRAY TRANSCRIPTION}

In this section we introduce Gray transcription and examine neutral evolution on using it. The method of transcription is to treat the codon as a binary string in Gray code. A codon is 
Gray coded if its adjacent integers on transcription are represented by bit strings that are Hamming distance 1 from each other.

Transcription is achieved by de-Graying the representation. What this involves is to find the binary value corresponding to the codon (which is in binary reflected Gray code), and then convert that binary value to an integer value. To convert Gray code to binary, we start with the most significant bit and sequentially update the string, with each bit being inverted if the next higher bit is set to one. For instance to convert 101 from Gray code to binary, the sequence is: $101 \rightarrow 111 \rightarrow 110$. Table 4 shows a set of 3-bit codons and their associated integer values on Gray transcription.

An exhaustive search for search neutral sets for derivation alternatives between 2 and 32, and codon sizes between 2 and 10 using Gray transcription and modulo translation showed that:

- When number of derivation alternatives $n_{i}=2^{k}$ we obtain the same number of search neutral sets as in the case of using natural transcription but the structure of the quotient graph is different (see examples in Fig. 8). In contrast to modulo transcription every single bit mutation leads to a change in derivation value. Thus for the case with two derivation alternatives we see the same behavior as a parity function for which every single transition results in a change of output.

- When number of derivation alternatives $n_{i} \neq 2^{k}$, no two codons are search neutral. Also the quotient graph shows that derivation neutral sets can be a Hamming distance of 1 apart (see example in Fig. 9. This puts a lower barrier to nontrivial neutral evolution.



Fig. 9. Sample quotient graphs for codons when using Gray transcription and modulo translation for 3 derivation alternatives with codon size of 3. Search neutral sets are vertices and connectivity between search neutral sets are directed edges. $[0 \mathrm{a}]=\left\{{ }^{\prime} 000 '\right\},[0 \mathrm{~b}]=\left\{{ }^{\prime} 010^{\prime}\right\},[0 \mathrm{c}]=\left\{{ }^{\prime} 101 '\right\},[1 \mathrm{a}]=$ $\left\{{ }^{\prime} 001 '\right\},[1 \mathrm{~b}]=\left\{{ }^{\prime} 110^{\prime}\right\},[1 \mathrm{c}]=\left\{{ }^{\prime} 100 ’\right\},[2 \mathrm{a}]=\left\{{ }^{\prime} 011^{\prime}\right\},[2 \mathrm{~b}]=\left\{{ }^{\prime} 111^{\prime}\right\}$.

It is apparent that for $n_{i} \neq 2^{k}$ nontrivial neutral evolution can occur even with single bit transitions and this arrangement means there is easier transition between codons representing different levels of robustness. In the alternative case of $n_{i}=2^{k}$ we see a low level of mutability with every single bit mutation event resulting in a change of the phenotype.

Table 5. Parameter values for experiments

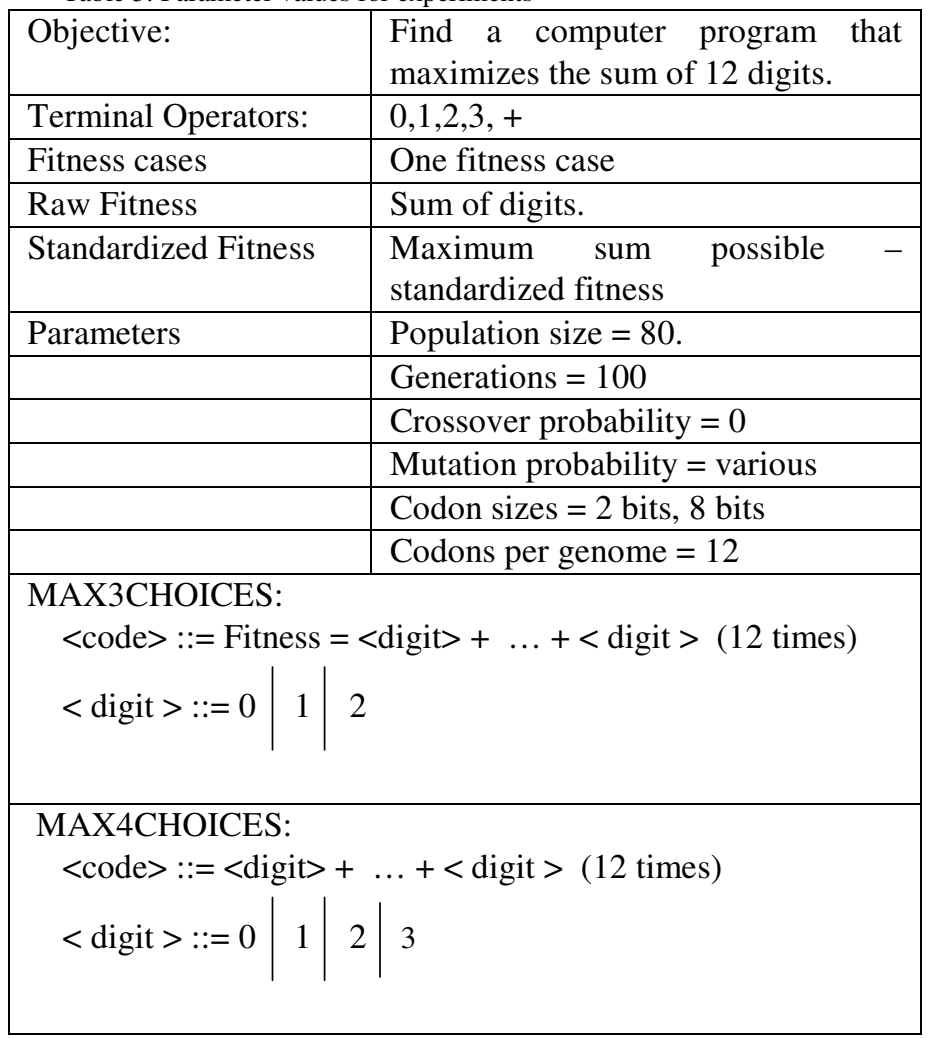

\section{EXPERIMENTS}

Our series of experiments are designed to validate some of the theoretical observations on transcription and translation models and degeneracy. These observations include:

- Some transcription/translation models give identical performance;

- Search being invariant to degeneracy when the number of rule alternatives is a power of 2 (using modulo translation);

The experiments involve using GE to find a computer program that maximizes the sum of 12 digits. Table 5 shows the two grammars used in the experiments. The grammar MAX3CHOICES has 3 derivation alternatives for each digit whereas for MAX4CHOICES the alternatives are a power of 2 (i.e. 4). For each grammar both natural and Gray transcriptions are tested in separate runs. All experiments used modulo translation. The codon sizes used were 2 and 8 bits. Fig. 10 shows an example of how a genome is converted to a program using the MAX3CHOICE grammar and 2 bit codons.

We have shown in the analysis (Section VII) that the order of derivation alternatives correspond to the use of different transcription models. In order to see how the ordering of the derivation alternatives in a GE system affects performance (and the invariance between some transcription models) all permutations of the digit alternatives were tested. This exhaustive test is also important because for GE, in general, 
there is no way of determining how to best order derivation alternatives without some prior knowledge. For MAX3CHOICES there are 3 ! ways of ordering the derivations of <digit>, all 6 such permutations were tested and are represented on the resulting plots. Likewise the 24 permutations for MAX4CHOICES were tested and are presented in the plots.

$01,11,10,00,10, \ldots$
Natural transcription
Fitness $=1+3,2,0,2, \ldots$
Program produced on modulo 3 translation

Fig. 10. Example conversion of a segment of genome to a program for a 2 bit codon size using the MAX3CHOICES grammar.

Each experiment involved 1000 replicates based off different random seeds. The parameter settings used for the experiments are shown in Table 5. For optimization we used the simple genetic algorithm with non-overlapping populations described in Goldberg [39]. We used roulette wheel selection and linear scaling, with no elitism. Success of an experimental run requires achieving the maximum possible fitness within 100 generations.

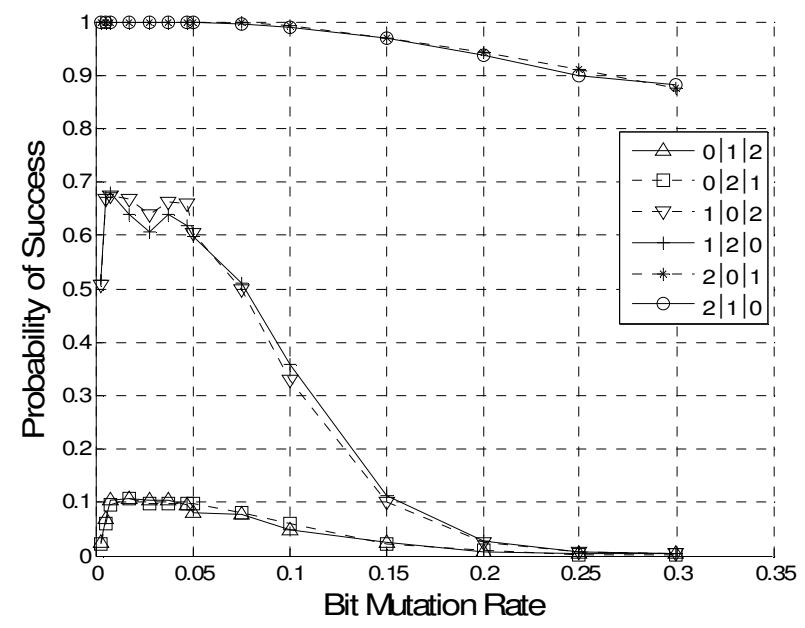

Fig. 11. Probability of success for 2-bit encodings using natural transcription with MAX3CHOICES. The legend shows the permutation of derivation alternatives being used.

\section{A. Experiment Results}

Fig. 11 shows the probability of success for 2-bit encodings using natural transcription with MAX3CHOICES. The graph legends indicate which of the six ways of ordering the derivation alternatives is being used. Fig. 11 shows the 3 classes of performance corresponding to the equivalent representations for different alternative orderings. This confirms that for either coding, the orderings $\{a|b| c\} \Leftrightarrow\{a|c| b\}$ are automorphisms.

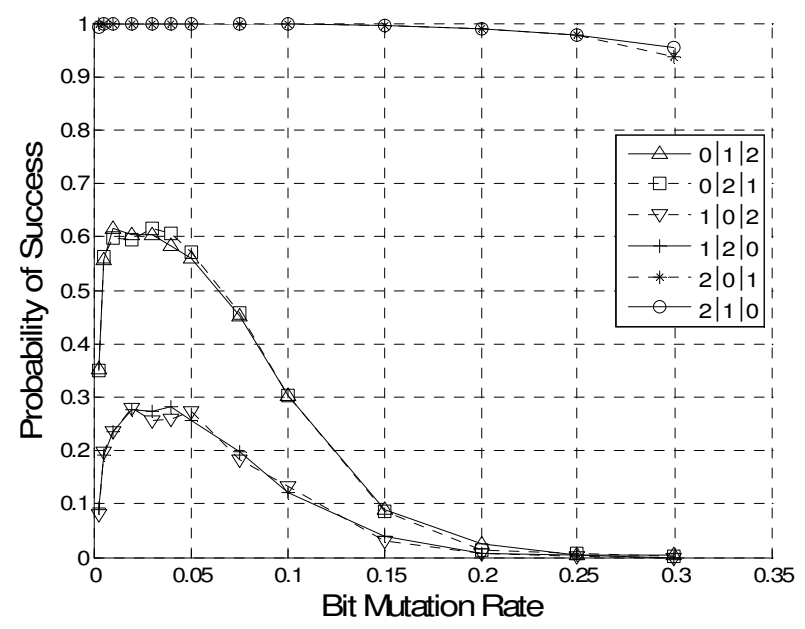

Fig. 12. Probability of success for 2-bit encodings using Gray transcription with MAX3CHOICES. The legend shows the permutation of derivation alternatives being used.

Fig. 12 shows the probability of success for 2-bit encodings using Gray transcription with MAX3CHOICES. Also noticeable from Fig. 12 are the 3 classes of performance corresponding to the equivalent representations for different alternative orderings. Fig. 13 shows the probability of success for 8-bit encodings using natural transcription with MAX3CHOICES. All six possible orderings of derivation alternatives are shown; statistical analysis of variances showed no significant differences between their performances. As a result there is not much point in putting a legend on Fig. 13. Fig. 14 shows the probability of success for 8-bit encodings using Gray transcription with MAX3CHOICES. All six possible orderings of derivation alternatives are shown.

We see from the graphs Fig. 11 to Fig. 14 that representation bias (i.e. an uneven representation of derivation alternative by codons) is an important factor when using low levels of degeneracy, but not as important with high levels of degeneracy.

Comparing the 8-bit and nondegenerate 2-bit codings on Fig. 15 and Fig. 16 confirms that with natural transcription and modulo translation, increased degeneracy does not affect performance when number of derivation alternatives is a power of two. Also evident in both plots are the 3 classes of performance corresponding to the equivalent representations for different alternative orderings.

We used data at the mutation rate of 0.025 to establish statistical significance. Our overall null hypothesis is that the order has no effect on probability of success. We conducted an analysis of variance based on 33 probabilities of success for each order; each probability of success is the success rate based on 30 randomly selected (without replacement) replicates for the relevant order. We used Fisher's Least 
Significant Method by first testing the overall null hypothesis for significance at the $95 \%$ confidence level. On finding significance (and rejecting the null hypothesis) we then compared all means pairwise using a t-test set at the same confidence level. We confirmed the following results:

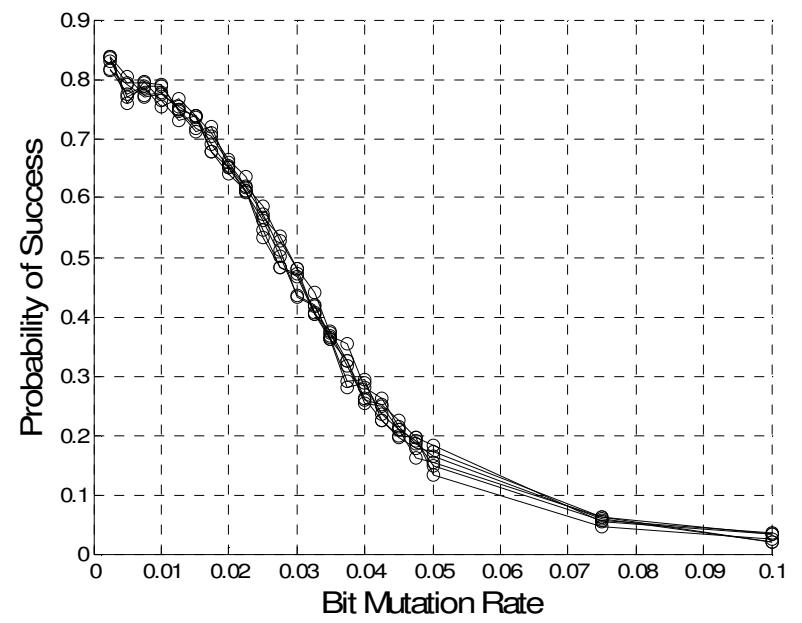

Fig. 13. Probability of success for 8-bit encodings using natural transcription with MAX3CHOICES. All 6 permutations of derivation alternatives are included.

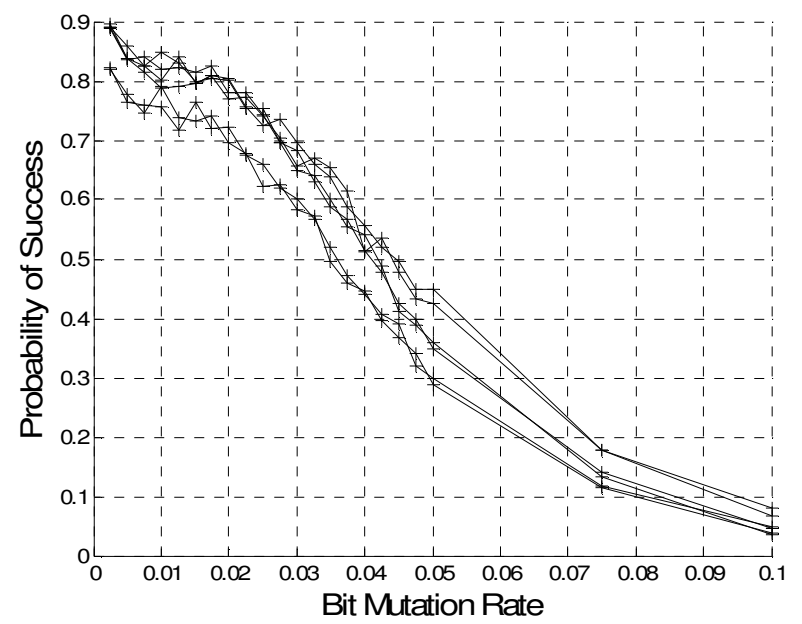

Fig. 14. Probability of success for 8-bit encodings using Gray transcription with MAX3CHOICES. All 6 permutations of derivation alternatives are included.

- There are statistically significant differences between orderings that are not automorphisms.

- There are no statistically significant differences between orderings that are automorphisms.

- Differences between the 2-bit and 8-bit coding are not statistically significant.

Fig. 17 and Fig. 18 show the probabilities of success for 8-bit and 2-bit encodings using Gray transcription with MAX4CHOICES. For the 8-bit encoding we see 6 classes of performance corresponding to equivalent representations. For the 2-bit encoding we see 3 classes of performance corresponding to equivalent representations.

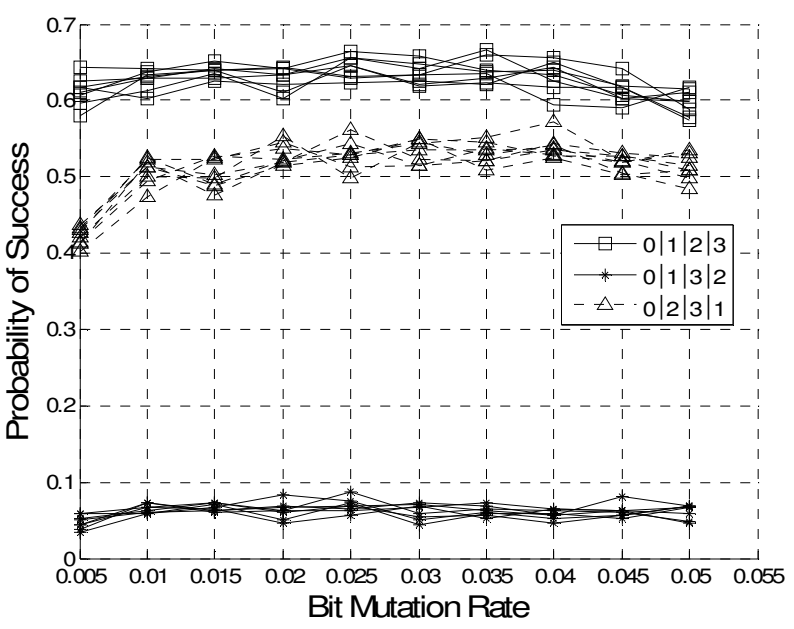

Fig. 15. Probability of success for 8-bit encodings using natural transcription with MAX4CHOICES. All 24 permutations of derivation alternatives are included.

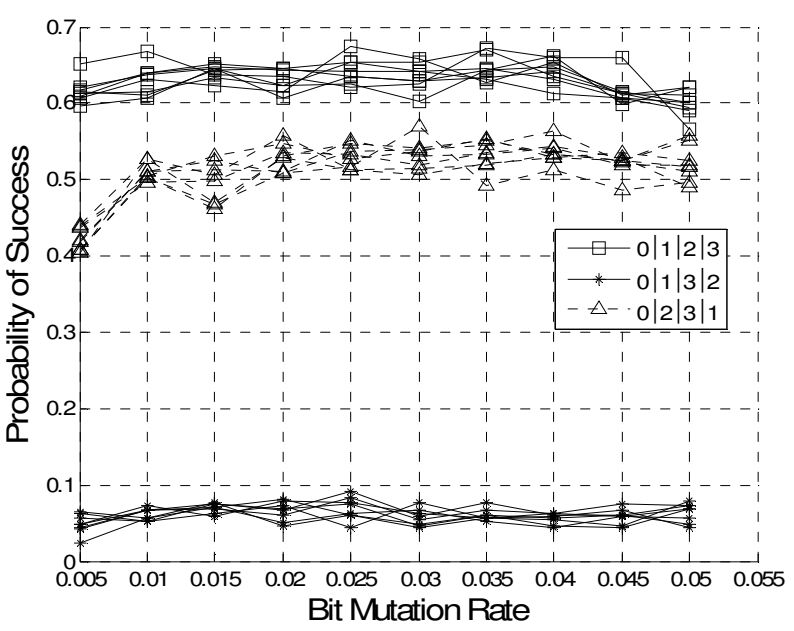

Fig. 16. Probability of success for 2-bit encodings using natural transcription with MAX4CHOICES. All 24 permutations of derivation alternatives are included.

In conclusion, we have validated the theoretical findings of transcription and translation invariance, as well as the fact that search is invariant to degeneracy when the number of rule alternatives is a power of 2 (for modulo translation).

\section{ANALYSIS OF RELATED WORKS}

This section starts with describing previous work by researchers in the areas of Neutral Evolution in Evolutionary Computing. In the first subsection we report the mixed results researchers have encountered on using degeneracy. We analyze the results of some related works based on our findings. Our focus is on works simple enough to be amenable to analysis and on addressing the conflicting results in the literature. Subsection B looks at prior work on Neutral Evolution in GE. The final subsection relates our quotient model with prior models for evolutionary systems. 
In order to not interrupt the flow of discussions in this section, details of problems and encodings used to support analyses are specified in the appendix.

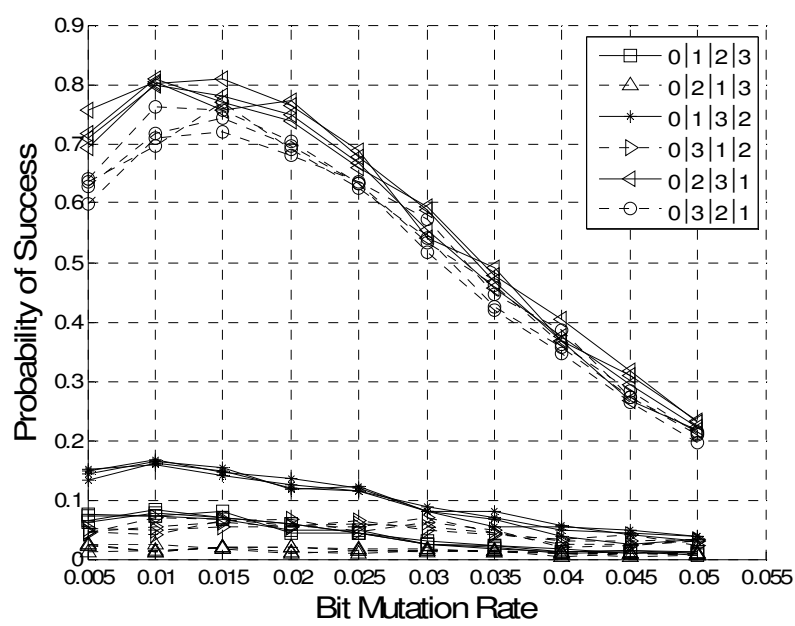

Fig. 17. Probability of success for 8-bit encodings using Gray transcription with MAX4CHOICES. All 24 permutations of derivation alternatives are included.

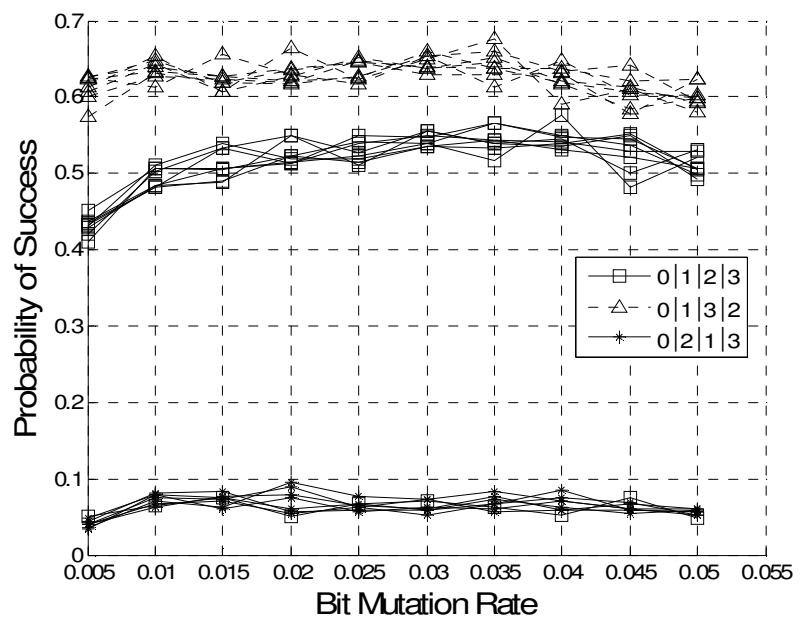

Fig. 18. Probability of success for 2-bit encodings using Gray transcription with MAX4CHOICES. All 24 permutations of derivation alternatives are included.

\section{A. Neutral Evolution}

Biological studies (e.g. [15], [40], [41]) have suggested that neutrality plays some role in evolutionary search, as a result the Evolutionary Computing community has been interested in analyzing and utilizing its effects. The majority of works have been devoted to exploring the relation between using some degenerate code and performance. Degenerate code have been reported to give better (or at least equal) performance to nondegenerate codes in many works; these include Harvey and Thompson [42], Julstrom [43], Banzhaf [44], Keller and Banzhaf [45], Yu and Miller [46, 63], Shackleton et al. [47], Shipman et al. [66] and Ebner et al. [67]. In such cases of performance improvement it is usually argued that using a degenerate code helped in avoiding premature convergence and allows escape from local optima on saddle surfaces.

Some works have found using a degenerate code caused a deterioration of performance; these include Davis [48], Eschelman and Schaffer [49], Ronald et al. [50], Smith et al. [51,52], and Knowles and Watson [68]. Reasons advanced for low performance include loss of genetic diversity, different genotypes that represent the same phenotype competing against each other and a larger search space size. In order to analyze the relationship between fitness and using a degenerate encoding we will first look at the association between fitness and form. A simple experiment that we will start with is that of Thomason and Soule [53].

\section{1) Fitness and Form}

Using simple experiments Thomason and Soule [53] showed that evolutionary systems can avoid fitter solutions in favor of solutions that are more robust to mutational change. The details of the experiment are explained in the appendix. Quotient graphs related to the experiments are also shown in the appendix (i.e. Fig. 20(i) and (j)). The graphs visually present how the mutational drives correlate in the direction of higher mutational robustness. The significance of the mutation rate on this drive can also be inferred from the graphs.

The result of Thomason and Soule buttress earlier empirical findings (such as those of Wilke et al. [54] ), and the quasispecies theory [55] that selection favors the cloud of genotypes that are interconnected by mutation whose average replication rate (i.e. fitness) is highest, rather than any one genotype that has the highest fitness. It can be argued that these results are related to the steady state distributions of evolutionary systems and, as such, have limited value to EC where the objective is to efficiently find a solution. However many evolving populations encounter periods of stasis (i.e. generations with no increase in fitness) prior to finding a solution. Such populations will, in varying degrees, (depending on how long they spend in stasis) adopt a steady state distribution among the suboptimal networks they have encountered. Consequently these distributions do factor into the efficiency of EC on non-trivial problems.

Although the experiments of Thomason and Soule [53] (and those of Wilke et al. [54]) pit robustness directly against fitness, these two attributes do not necessarily have to be in contention. We can, for instance, reassign fitness such that the fitness drive is in the same direction as the mutational drive. However in the usual practice of EC, the objective is usually to find a single (or relatively rarely occurring) solution in a nontrivially large representation space. As a result of being rare, the solution usually do not have the alternative genotype forms that are necessary for making a correlated mutational drive point in their direction.

Instead of considering a population of two competing phenotypes, we can generalize the result of the above experiments into a population of multiple competing phenotypes. There are three factors that can affect each phenotype's performance in such a competition; these are its 
relative fitness, its relative invariance to mutation (which is related to the number of alternative forms it can take without having its fitness altered), and its neighborhood (i.e. the fitness of its search distribution). All of the factors are represented on quotient models. The importance of the various factors will vary depending on the mutation rate. An important question is how does using a degenerate code alter the factors.

A set of experiments that can help answer that question is that of Poli and Galvan [56]. Poli and Galvan [56] explored three different genotype-phenotype mappings in their study of how fitness distance correlation of landscapes change under the effect of different neutral encodings. These were majority, parity and truth table coding. Using the formalism proposed by Toussaint [57], they exemplified trivial neutrality with mapping based on parity encoding. Examples quotient graphs of both majority and parity coding are given in the appendix (see Fig. 20(e), (f) and (g)). They also introduced the concept of a phenotypic mutation rate. The phenotypic mutation rate of a code (at a particular bit mutation rate) is the expected probability of a change in its phenotype value on mutation. In terms of the quotient model, the phenotypic mutation rate is the sum of all elements of the non-neutral quotient matrix, $\bar{M}_{\text {non-neutral }}$ of equation (1) (specified in Section IV (C)). This phenotypic mutation rate is a summary value on discarding all components of variation that do not directly contributes to fitness change. Our opinion as to why the phenotypic mutation rate was shown to be a good performance predictor is that the performance of the three types of degenerate encodings tested is more consistent when their different topologies are accounted for by discarding the elements of search that do not lead to fitness change.

Other ways in which evolutionary systems evolve better robustness include the evolution of compact representations in grammar-based coding [58], bloat in genetic programming reducing the probability of deleterious crossovers [59 ], and canalization [60] (i.e. reducing the number of inputs that can affect outputs) for binary genetic networks that adapt the genotype-to-phenotype map. We now go on to look at other dynamical attributes of neutral evolution.

\section{2) Evolvability, Connectivity and Tunneling}

Neutral networks are usually attributed with increasing evolvability, connectivity and the enabling of genomes to tunnel through portals to higher fitness, and escape local optima through high-dimensional saddle surfaces. The main experiments we will use to examine these attributes are those of Galvan and Poli [18], [61].

Galvan and Poli $[18,61]$ stated some reasons, with which we agree, on why results on neutrality are mixed. These include:

- Conclusions being based on performance statistics rather than analyses of population dynamics.

- Studies being based on relatively complex problems leading to results that are the effects of many inseparable factors.

- Different definitions and approaches to neutrality.
- The features of a problem can change with adding neutrality.

They concluded that it is very difficult to infer the effects (or benefits) of neutrality without analyzing population flows induced by the presence of neutrality. They therefore analyzed population flows for both a modified OneMax and a modified deceptive trap problem. Both problems were adapted such that there is an additional bit in the genotype that signifies whether the phenotype is on the problem's network or on a neutral network of constant fitness.

In the appendix (Fig. 21(c)), we show the quotient graph for a 4-bit version of the OneMax problem adapted in the same fashion as in [18] and [61]; Fig. 21(d) details the fitness drives (with solid arrows) and correlated mutational drives (with dotted arrows) that explain the population movements on such networks. Fig. 21(e) shows a similar graph for the modified deceptive trap problem. Even though the problems and encodings in [18] and [61] are chosen based on their simplicity, we observe from Fig. 21(d) and (e) that there is a compound effect of drives in multiple directions; this results in the complex movements of populations on such landscapes as observed by the authors. Fig. 21(c) shows that for this 4-bit problem there is a $4: 1$ ratio of a genome staying in a layer rather than transiting between neutral and adaptive layers on single bit mutation. The neutral layer is canalized in the sense that only a single bit position can possibly affect the fitness of any genome on that layer.

By inspection of the mutational drives for both problems, we expect net population movement on the neutral layer to be towards the central node (i.e. the node labeled [3d]) of that layer. Although node [3d] is equally as fit as the rest of the neutral layer, from the quotient graph point of view it is a local optima. It has a basin of attraction (i.e. the rest of the neutral layer), and it is locally optimal in the sense of being preferred by selection (for its robustness).

Galvan and Poli $[18,61]$ showed that the availability of the neutral layer led to a deterioration of performance. This is not surprising considering the above discussion on the drives and local optima, however the said experiment demonstrates many concepts that have been used to explain evolutionary behavior in the presence of neutrality, including percolation, tunneling, and escape from local optima on saddle surfaces. Percolation [62] is the condition where some neutral network is spread throughout a search space in such a way that no genotype is further than a certain distance from it. Every genotype is at most Hamming distance 1 from the neutral networks of the modified OneMax and deceptive trap problems. A neutral escape path from the local optima [3a] of Fig. 21(e) is the path sequence [3a] $\rightarrow[3 \mathrm{~b}] \rightarrow[3 \mathrm{c}] \rightarrow \ldots \rightarrow[3 \mathrm{f}] \rightarrow[4]$. Note however that the net drive is no longer in the direction of the path after reaching the local optima [3d]. In general the dynamic of a genome in [3d] is more likely to hover in the neighborhood of that node than to proceed to the global optimum.

The results of the experiments of Poli and Galvan show that having neutral pathways and high fitness percolating networks do not guarantee an improvement in performance. They also demonstrate the complicated population dynamics relatively simple evolutionary systems can undergo. We believe 
explaining the dynamics would be difficult without accounting for the "forces" that drive the population movements. Correlated mutational drives, which are due to the topology of networks, are an important part of such explanations.

\section{3) Reduced Boolean Parity on CGP}

$\mathrm{Yu}$ and Miller [63] applied Cartesian Genetic Programming (CGP) to a simplified form of the Boolean parity problem. This reduced form uses only EQ and XOR (i.e. the equality and "exclusive or" functions) as part of its function set. Yu and Miller [63] reported that the neutral networks used by utilizing a $(1+4)$ evolutionary strategy (i.e. with one parent spawning 4 children, and fittest or equally fit child becoming parent for next generation) improved, or at least did not deteriorate search performance. Collins [64] however reported that sampling biases inherent in the CGP representation make the CGP search strategy perform worse than either randomly sampling the reduced Boolean parity formula space, or randomly sampling a CGP-like representation that does not allow introns.

In the appendix (Fig. 21(a) and (b)), we show quotient graphs for the CGP implementation of a 2-parity problem using only two gates and the equality (EQ) function. This trivial 2-parity problem is adequate to illustrate some issues behind the conflict. The conflict is itself based on a 12-parity, 100 gates problem using the EQ function.

In CGP the output is taken from a fixed output node, which is the last node in the genome. Collins however used an algorithm where the output is taken from a randomly selected node. Fig. 21(a) and (b) corresponds to the CGP approach and Collins' approach respectively. We see that the genotype space and search dynamics are different, based on the approach being used; this questions the validity of comparisons based on using such different algorithms.

Our interest is to explain CGP performance on this problem in terms of neutral evolution. To confirm whether the CGP (1 +4 ) evolutionary strategy improved performance relative to random search of the CGP representation for the reduced 12parity problem using 100 gates, we randomly sampled the CGP representation space. The MATLAB ${ }^{\circledR}$ code used for both the CGP $(1+4)$ and random search are available for download at [65]. We obtained 751 solutions based on 50 million sampled genomes. This puts the probability of finding a solution on randomly evaluating 40000 genomes at $45 \%$. This rate was not equaled or exceeded by using the CGP $(1+$ 4) algorithm. We obtained a success rate of $40 \%$ based on 500 runs of evaluating of the CGP $(1+4)$ algorithm for 10000 generations at $20 \%$ mutation rate for instance. Our conclusion is that the algorithm does not perform better than random search on this problem.

Of interest is the fact that there are only two fitness levels for the reduced Boolean parity problem; the global optimum, and a common fitness for all other genomes. There is therefore no fitness gradient to guide any search algorithm while an optimum has not been found. There is also no selective advantage for robustness, as all the suboptimal genomes are equally robust (there not being a less fit set of genomes to evolve away from). The reason why the CGP $(1+4)$ controlled random walk does not perform as well as random search on this problem is due to locality issues. As Collins [64] noted the solutions are not randomly distributed. Also the inputs to any set of gates not used in a particular solution can be changed, and the resulting genome will still be a solution. What this means is that solutions are clustered in genotype space. If a genome is a solution, there is an above average probability of its neighbors being solutions; similarly there is a below average probability of a genomes neighbors being solutions if it is not itself a solution. This accounts for the poorer performance of the localized random walk.

\section{4) Random Boolean Networks}

Shipman et al. [66] used a random Boolean network (RBN) mapping that yields a very high degree of degeneracy and showed that this caused the discovery of many more phenotypes than would be the case with a coding without redundancy. This increased accessibility was found to significantly aid evolutionary search on a fitness-adaptive walk. They explained that the presence of beneficial neutrality aided the search process in a telecommunications application.

Ebner et al. [67] compared non-redundant binary mapping with a similar RBN map as Shipman et al. [66], and with highly redundant Cellular Automata (CA). Ebner et al. [67] showed that evolvability (which they defined as the ability of random variations to sometimes produce improvement), is influenced by the existence of neutral networks in genotype space. They demonstrated that redundant mappings allow the population to spread along the network of neutral mutations and the population is able to recover after a change has occurred. They also explained that the population can sustain high mutation rates.

Knowles and Watson [68] also used RBN maps. They however did not report performance improvement for the redundant RBN map when compared to a non-redundant encoding. The results obtained by Ebner et al. [67] and those obtained by Knowles and Watson [68] are not directly comparable as the former applied a hill-climbing algorithm based on single mutations to genomes and the latter used a per bit mutation rate that can result in multiple bit changes on a genome.

A common problem used in [67] and [68] is the "hierarchical if and only if" (H-IFF) function. Ebner et al. [67] found a performance improvement on using the highly redundant RBN map on a 16 bit H-IFF function (compared to a non-redundant coding). In the appendix (Fig. 23) we show the quotient graph of the 8 bit H-IFF function. Fig. 23 shows the local optima that are likely to trap genomes using a hillclimbing algorithm.

With bitwise encoding of phenotypes (such as parity or majority coding), a single bit change of a genome can result in one bit change of the phenotype at most. With Encodings based on CA and RBN however a single bit change of the genome can result in multiple changes to the phenotype. These non-bitwise encodings can result in changes of a problems 
landscape similar to using a different representation (with or without degeneracy).

\section{B. Grammatical Evolution}

In the case of GE the application of neutral evolution is achieved by making use of a degenerate genetic code [5], [6], [7]. The degenerate code is presumed to increase the occurrence of neutral mutations within a population.

O'Neill and Ryan [69], [7], conducted a study on the effect of degeneracy on a symbolic regression problem and the Santa-Fe Trail problem. The syntax of the grammar used for the symbolic regression problem is an instance where the number of derivation alternatives for every production is a power of 2 . We have shown that for such grammars the presence of degeneracy does not have any effect on performance, and the additional genetic material on increasing degeneracy does not affect the GE search process. This is consistent with the authors [7], not finding the variation of performance with degeneracy conclusive for the first 20 generations on using the symbolic regression grammar.

The grammar for the Santa-Fe Trail problem is an instance where the number of derivation alternatives for every production is not a power of 2 (as shown in Fig. 1). O'Neill and Ryan [69], [7], showed an increase in performance on using a degenerate (i.e. 8 bit) coding as opposed to using a nondegenerate (i.e. 2 bit) coding. We have experimented with the specific representational and evolutionary biases involved in the Santa Fe Trail problem and the results will be published elsewhere.

In O'Neill et al. [70] some modification was made to the Santa-Fe ant grammar by adding explicit introns. On adding an intron to the last production of the Santa-Fe ant grammar (see Fig. 1), the number of derivation alternatives for every production becomes a power of 2 . The results for the degenerate and non-degenerate coding in that case can be compared by visual assessment of the plots in [70]. These are consistent with our findings of equal performance.

Nicolau [71] investigated the effect of grammar size and complexity on performance. A method to reduce the number of non-terminal symbols in a grammar was presented. The method tries to maintain the bias of the original grammar by maintaining the ratio of possible derivations, however the effects of the mod rule are not accounted for. This method was applied to four problem instances and non-statistically significant improvements were reported.

Rothlauf and Oetzel [72] investigated the locality of the genotype-phenotype mapping (representation) used in grammatical evolution (GE). They showed that many neighboring genotypes do not correspond to neighboring phenotypes. In experiments with a simple local search strategy, the GE representation lead to lower performance for mutation based search approaches in comparison to standard GP representations.

Other works include GE variants like piGrammatical Evolution [73] that uses a position-independent variation of the mapping process. Another variant is the meta-Grammar
Genetic Algorithm [74] that uses a universal grammar to derive a solution grammar and then derive a solution.

\section{Models of Evolutionary Computing}

There have been many approaches at modeling evolutionary phenomena starting with the schema theorem [75] that predicts the growth of schemata with above average fitness. Vose [76] and Radcliffe [77] established that any subset of the search space (i.e. not just schemata) satisfied the schema theorem. They subsequently independently generalized schemata into predicates (which are classes of genotype that correspond to the truth of some specified condition) and formae (which are classes based on equivalent sets of genotypes) respectively. Predicates were subsequently used to study transformations of binary representations and genetic operators that reduce problem difficulty. Similarly formae were used to develop and formalize binary and non-binary representations and operators that lead to improvement in search performance $[78,79,80]$. Both predicates and formae being generalizations of schemata do not, in general, make exact predictions about evolutionary systems. Also the choice of a particular schema, predicate or forma for a particular analysis is chosen based on its perceived usefulness. A guiding principle for such usefulness is that the fitness variance between genetic forms that correspond to the same (or similar) phenotype should be low.

Rothlauf and Goldberg [81] also made a distinction that is based on the stated low fitness variance principle. Rothlauf and Goldberg [81] classified representations into synonymously and nonsynonymously redundant groups. Representations are synonymously redundant if the genotypes that represent the same phenotype are very similar to each other. Similarity is measured by applying a distance metric on the genotype space. Synonymously and nonsynonymously redundant representations lead to good and poor search performances respectively. Some papers (e.g. [82]) have successfully used the concept of transforming a nonsynonymously redundant representation in order to improve search. Rothlauf and Goldberg used the trivial voting map [47, 81] as an example of a synonymously redundant representation. Details and a quotient graph of this map are shown in the appendix (see Fig. 20(b)).

\begin{tabular}{cccc}
\hline genotype & Fitness & \\
\hline 00 & 0 & \\
01 & 0 & 00.110 \\
10 & 5 & 100 \\
11 & 0 & \\
\hline
\end{tabular}

Fig. 19: genotype-fitness map and quotient graph of a 2-bit example

Another model that has been found useful in EC is that based on unitation. Unitation classes are defined by the number of ones in the genotype, and not by the positions the ones occupy. Like schemata, all unitation classes conform to some predicate and are equivalence classes; unitation classes 
are therefore subsets of predicates and formae. Examples of unitation functions (i.e. functions that map unitation classes to the same phenotype) in the appendix are Fig. 20(e), (f) and (g).

The Quotient models introduced in this work are unique in their explicit use of search distributions in their definition. Quotient models are exact Markov models that represent the genotype-to-phenotype map. We end this subsection by illustrating the similarities and differences between various model classes by use of an example. Fig. 19 is an example of a 2-bit genotype to fitness map and its associated quotient graph. Table 6 classifies some subsets of this map according to various model classes. Table 6 shows, for instance, that " 00 " and " 11 ", considered together as a set, do not correspond to any schemata or unitation class. They correspond to the formae representing the equivalence class of genotypes with all their bits identical. They also correspond to the predicate of all genotype that meet the condition of all bits being equal. They are not synonymously redundant because they are not genetically similar (having the maximum Hamming distance of 2 between them). The genotypes are search neutral because they have identical fitness and search distributions.

Table 6: Classification of some subsets of the genotype of Fig. 19

\begin{tabular}{|c|c|c|c|c|c|c|}
\hline 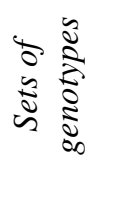 & 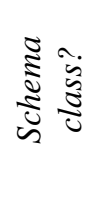 & 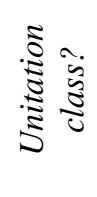 & 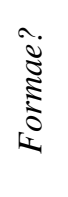 & 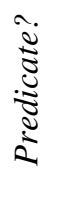 & 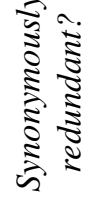 & 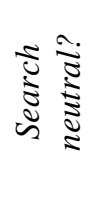 \\
\hline$\{00,11\}$ & no & no & yes & yes & no & yes \\
\hline$\{01,11\}$ & yes & no & yes & yes & yes & no \\
\hline$\{01,10\}$ & no & yes & yes & yes & no & no \\
\hline
\end{tabular}

\section{CONCLUSIONS AND FUTURE WORK}

In analyzing how mapping (including degeneracy in maps) influences search and neutral evolution we have formalized a procedure for modeling search by distilling the interaction between representation and search into quotient sets and graphs. Quotient models are applicable to degenerate maps. They take advantage of the symmetries inherent in many mapping schemes to give exact models of the evolutionary process; thereby they make understanding evolution easier by showing only what is important, and in a reduced representation. We have shown that they reduce the degrees of freedom required for modeling genes and genomes in many cases.

Our use of quotient graphs allowed us to reduce the effective degrees of freedom required to understand GE mapping without resorting to an approximate model, and in the case of the codon, visualizing search distributions and symmetries. Quotient graphs enable us to predict when increasing codon size or permuting certain right hand terms will not affect search performance. This work puts a new perspective on diversity in GE populations as we identify genomes that have codons in different states but are virtually identical in their fitness and search distributions.

In GE genotype-to-phenotype maps are composed by combining a transcription and a translation model. We have examined the attributes of natural and Gray transcription, as well as modulo translation. Having shown that there are equivalence relationships between some transcription models means that we have, in effect, looked at the behavior of classes of such models. We derived the size and structure of the model class; the model class of transcription models can be formed by the application of bit-masks and / or permutations on a member of that class.

Although this work is primarily concerned with GE, it should be noted that variants of GE including Genetic Algorithms using Grammatical Evolution (GAUGE) [83], meta-Grammar Genetic Algorithm [74], Christiansen Grammatical Evolution [84] can use natural transcription and modulo translation model as part of their genotype to mapping process, therefore this work is relevant to them as well.

It has been shown that for natural transcription used with modulo translation, there is a significant barrier to nontrivial neutral evolution. This finding is based on our proving a necessary but not sufficient condition of at least 3 bit transitions within a single codon for nontrivial neutral change. This result is independent of the level of degeneracy of the coding. It was shown that use of Gray transcription with modulo translation can lower this barrier. This combination can allow nontrivial neutral evolution with single bit transitions.

For natural transcription and modulo translation, when the number of derivation alternatives is a power of 2 , neutrality is trivial and search is unaffected by degeneracy. This was shown analytically and confirmed empirically. It was also empirically shown that the ordering of derivation alternatives can have a significant effect on performance.

We presented a practical basis for the classification of neutrality in Evolutionary Computing. This classification includes a syntactic class (which has to do with the encoding in use) and a semantic class (that is inherent to the problem at hand). The theoretical analysis in this paper is mainly based on syntactic neutrality.

Whether adding degeneracy to a map will help, hinder or leave a search process unaffected will depend on how such degeneracy alters genetic neighborhoods. Degeneracy can rearrange a map such that random bit mutations are correlated to create an evolutionary drive that is distinct from the drive due to fitness. This unsuspecting evolutionary bias is all the more important considering the penchant for using maps that are highly structured in Evolutionary Computing. Another effect of adding degeneracy is that it can allow neutral transitions between genes allowing them to identify islands of robustness. We have seen however that in some cases neutral members cannot easily transition between themselves, and in some cases, the degenerate search space can be projected into 
a subspace with the same neighborhood structure as a nondegenerate search space, meaning that degeneracy added nothing to the search process.

Future work will include searching for mapping schemes that give good performance under a range of different conditions. We will also be looking at Evolutionary Dynamics in GE in order to further characterize search behavior especially with regard to crossover and wrapping. Further work will also include a comprehensive look at semantic forms of neutrality and the interaction between syntactic and semantic neutrality.

\section{APPENDIX}

The encodings, functions and graphs in this appendix are to:

- Show the wide applicability of quotient graphs;

- Support and illustrate the analysis of related works.

\section{A. Codes and Functions of Unitation}

Fig. 20 and Fig. 21 show examples of quotient graphs for some encodings and problems that have been used in the EC literature. Fig. 20 (c), (d) and (h) are examples on how they can be generalized to the n-bit case for majority and parity encodings. Rather than drawing multiple arcs between nodes, it is sometimes more convenient to indicate the number of arcs by a number beside a single arc; this is done for Fig. 20 (c), (d), (h) and (j).

Majority coding works as follows: given $\mathrm{n}$ bits (assume $\mathrm{n}$ is odd so there cannot be an equal number of zeros and ones in a gene), If the number of ones is greater than the number of zeros then the phenotype level is set to 1 , otherwise it is set to 0 . For the 3-bit majority coding of Fig. 20 (a), [0a] $=\{000\}$, $[0 \mathrm{~b}]=\{001,010,100\} .[1 \mathrm{a}]=\{011,101,110\},[1 \mathrm{~b}]=\{111\}$.

For majority coding with $\mathrm{n}$ even, we obtain the Trivial Voting map that has been used in [47] and [81] and [85]. Fig. 20 (b) shows 4-bit majority coding. This map works the same way as in the case for $\mathrm{n}$ being odd if the number of zeros and ones are not equal. When the number of zeros and ones are equal, the phenotype is set to the value of the leftmost bit. Note the similarity of Fig. 20 (b) to the 4 bit, 2 derivation alternatives map of Fig. 7; both maps show trivial neutrality. As noted by Rothlauf [85], the even-sized majority encoding does not affect search performance.

Parity coding works as follows: if the number of ones that are in $\mathrm{n}$ genotypic bits is an even number, then the bit at the phenotype level is set to 1 , otherwise it is set to 0 . For the parity coding of Fig. 20(c), [0] is the set of all n-bit strings with even parity, [1] is the set of n-bit strings with odd parity.

Needle in haystack (NIH) problem: there is a single genome with optimal fitness of 1 . All other genomes have the same suboptimal fitness 0 . For the 3-bit Needle in haystack genomes of Fig. 20(d), [1] $=\{111\},[0 \mathrm{a}]=\{000\},[0 \mathrm{~b}]=$ $\{011,101,110\},[0 \mathrm{c}]=\{001,010,100\}$.

Deceptive trap problem: The fitness is the number of ones in the genome. However if there is no " 1 " in the genome the fitness is $n+1$ for an $n$-bit genome. For the 3-bit deceptive trap of Fig. 20(e), [3] $=\{111\},[4]=\{000\},[2]=$ $\{011,101,110\},[1]=\{001,010,100\}$.

OneMax problem: In the case of Fig. 20(f), the fitness is the number of " 1 " in the genome. For the 3-bit OneMax coding of Fig. 20(f), [3] $=\{111\},[0]=\{000\},[2]=\{011,101,110\},[1]$ $=\{001,010,100\}$.

Fig. 20(g) shows the OneMax problem implemented with parity encoding. The fitness in this case is the sum of the fitness of the 3 genes that compose the genome. These genes are n-bit strings that are parity encoded.

The NIH, Deceptive trap and OneMax problems are examples of functions of unitation. Functions of unitation have their phenotypes defined only by the number of ones in the genotype, and not by the positions the ones occupy [86]. It can be seen that for such functions, the mutational drive is from the edge nodes towards the central nodes. The NIH, Deceptive trap and OneMax problem have an edge node as their fittest node. It has been shown for these and other functions of unitation (Ritchie et al. [87]) that on increasing the mutation rate the steady state distribution of a population of genes (i.e. the quasispecies) moves from a population composed almost entirely of the fittest gene (which is an edge node of the quotient graphs) to one composed increasingly of what is towards the centre of the quotient graphs. This change of distribution is due to the correlated mutational drive becoming more dominant with increased mutation rate.

\section{B. Thomason and Soule [53]}

Fig. 20(i) and (j) are based on a redundant coding scheme used by Thomason and Soule [53]. Fig. 20(i) shows the quotient graph of the coding scheme and Fig. 20(j) shows the quotient graph of a genome composed of 3 such genes.

The gene of Fig. 20(i) is a one of four possible characters $\{\mathrm{A}, \mathrm{X}, \mathrm{Y}$ or $\mathrm{Z}\}$. On mutation any gene can change into itself or any other gene with equal probability. Note that $\bar{A}=\{X, Y, Z\}$.

For Fig. 20(j), if the count of $A$ 's in a genome is larger than the count of all other characters combined, then the fitness of the genome is equal to the count of $A$ 's. Otherwise the fitness is the length of the string minus the $A$ count. The goal is to obtain a string of either all $A$ 's or no $A$. For Fig. 20(j), $\bar{A} \bar{A} \bar{A}, A \bar{A} \bar{A}, A A \bar{A}$ and $A A A$ represents the $27,27,9$ and 1 possible genomes with no $A$, one $A$, two $A$ and three $A$ 's respectively. These genomes have fitness of $3,2,2$ and 3 respectively.

The experiments of Thomason and Soule [53] show the effect of a net mutational drive. Each experiment started with the number of A's exactly matches the combined number of $X$, $\mathrm{Y}$ and Z's for all individuals. They used a weight factor that gave extra fitness to genomes composed of mostly A's, so as to see to what extent degeneracy was selected for more than fitness. 


\begin{tabular}{|c|c|c|c|c|c|c|c|c|c|}
\hline $\mathrm{a}$ & $\mathrm{b}$ & $\mathrm{c}$ & $\mathrm{d}$ & $\mathrm{e}$ & $\mathrm{f}$ & $\mathrm{g}$ & $\mathrm{h}$ & $\mathrm{i}$ & $\mathrm{j}$ \\
\hline 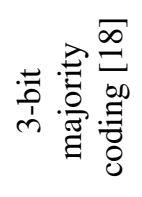 &  &  & 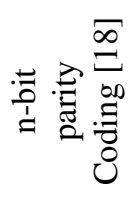 &  & 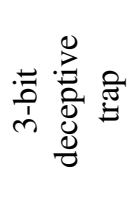 & 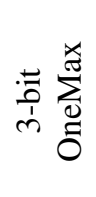 & 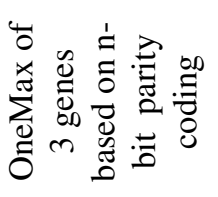 & 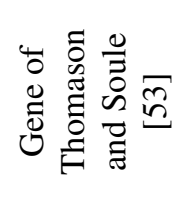 & 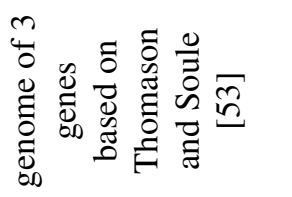 \\
\hline 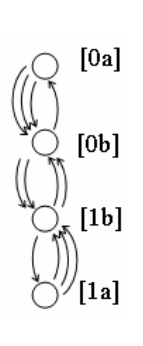 & & & $\begin{array}{c}{[0]} \\
0 \\
n()^{n} \\
0 \\
{[1]}\end{array}$ & 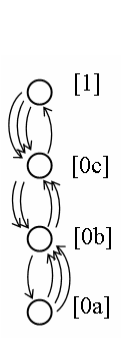 & 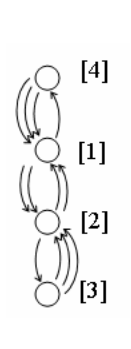 & 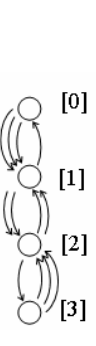 & & & 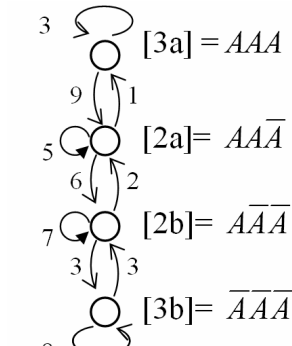 \\
\hline
\end{tabular}

Fig. 20: Sample quotient graphs for genes and genomes used in various coding schemes and problems.

\begin{tabular}{|c|c|c|c|c|}
\hline $\mathrm{a}$ & $\mathrm{b}$ & $\mathrm{c}$ & $\mathrm{d}$ & $\mathrm{e}$ \\
\hline 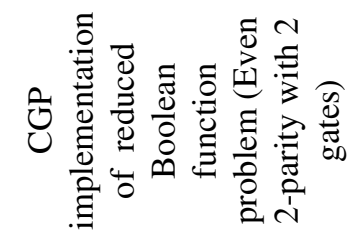 & 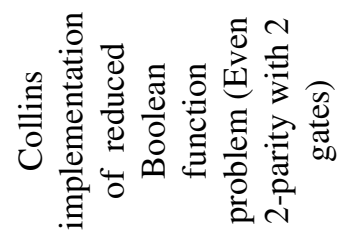 & 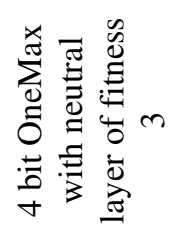 &  &  \\
\hline  & & [4] & [3] & ${ }_{[3 \mathrm{a}]}^{[4]}$ \\
\hline
\end{tabular}

Fig. 21: Sample quotient graphs for Even 2-parity, OneMax and deceptive trap with neutral layer 
Thomason and Soule used genomes composed of 100 genes, rather than the 3 genes example shown in Fig. 20(j). Our explanation applies for either case. We can see from Fig. 20(j) that the mutational drive towards $\bar{A} \bar{A} \bar{A}$ is higher than that towards $A A A$; consequently we expect the proportion of solutions of form $\bar{A} \bar{A} \bar{A}$ to be higher than that of the form $A A A$. We also expect the relative proportions to be dependent on the mutation rate, with the proportion of $\bar{A} \bar{A} \bar{A}$ solutions increasing with increased mutation rate. These are some of the findings of Thomason and Soule [53]; they conclude that the evolutionary system can avoid a more fit solution in favor of a more robust solution, when under pressure for robustness combined with function sets containing redundant genes.

\section{Cartesian Genetic Programming}

Fig. 21(a) shows the CGP implementation of a reduced Boolean function problem (Even 2-parity with 2 logic gates using only the Equality operator) $[63,64]$.

The CGP genotype is a string of integers. The genotypes are usually composed of sequences of integer triples, each of which represents a unit equivalent to a logic gate. One of the integers in the triple represents the gate type; because we are only going to use one gate type (i.e. the Equality (EQ) function) we are not going to represent the gate type in our genomes. Consequently our genomes are composed of a sequence of integer doubles. The two integers in a double represent the connections to either one of the 2 function inputs or the output of a previous gates. The output of the genome is taken from the final gate.

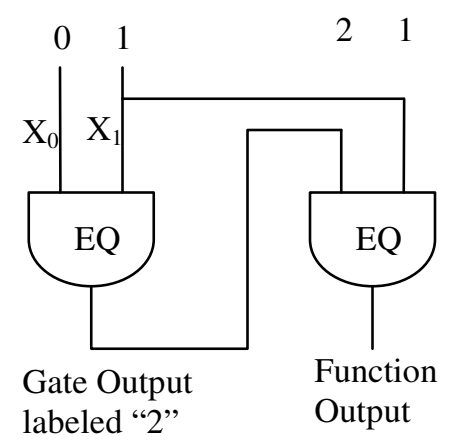

Fig. 22: The phenotype corresponding to genotype "01 21" for the CGP implementation of even 2-parity using 2 gates.

The two function inputs are labeled 0 and 1 on the genotype and $X_{0}$ and $X_{1}$ in the phenotype. Each gate has 2 inputs. The gates are labeled 2 and 3 in the genotype. The labels are used for referencing the function inputs and the outputs of the gates. Fig. 22 shows the phenotype for a CGP genotype "01,21". The first gate receives function inputs "0" and "1". The second gate receives the output of the first gate and function input
"1". It is possible for both inputs of the second gate to be function inputs; in that case the first gate does not affect the output. For the 2-parity, 2 gates problem there are 36 possible genomes spanning the range " 00,00 " to " 11,22 ". The solutions are the eight genomes that have the input to the second gate as " 01 " or " 10 ".

In CGP output is taken from the final gate (which is the second gate in our case) [63,88]. Collins however implemented algorithms wherein the output is taken from a randomly chosen gate. This increases the genotype space to 72 genomes for the 2-parity, 2 gates problem (i.e. the original 36 genomes for the output being taken from the second gate, and another 36 for the output being taken from the first gate). Additional solutions generated by taking output from the first gate are the 18 genomes having the first gates input as " 01 " or "10".

Note that CGP uses integer coding (i.e. on mutation, an allele can change into any other valid integer value with equal probability). This coding leads to highly connected quotient graphs as seen in Fig. 21(a) and (b). Table 7 and Table 8 show examples from the 36 and 72 possible genomes (and the quotient set they belong to) for the quotient graphs of Fig. 21(a) and (b). The dark nodes of the stated figures correspond to solutions.

Table 7: Examples of genomes that belong to the search neutral sets of Fig. 21(a).

\begin{tabular}{|l|cccc|}
\hline Label & \multicolumn{4}{|c|}{ genotype } \\
\hline $0 \mathrm{a}$ & 0 & 0 & 2 & 2 \\
\hline $0 \mathrm{~b}$ & 0 & 0 & 0 & 2 \\
\hline $0 \mathrm{c}$ & 0 & 0 & 0 & 0 \\
\hline 1 & 0 & 0 & 0 & 1 \\
\hline
\end{tabular}

Table 8: Examples of genomes that belong to the search neutral sets of Fig. 21(b).

\begin{tabular}{|l|llll|r|}
\hline Label & \multicolumn{3}{|c|}{ genotype } & $\begin{array}{r}\text { Output } \\
\text { taken from }\end{array}$ \\
\hline $0 \mathrm{a}$ & 0 & 0 & 2 & 2 & gate 2 \\
\hline $0 \mathrm{~b}$ & 0 & 0 & 0 & 2 & gate 2 \\
\hline $0 \mathrm{c}$ & 0 & 1 & 2 & 2 & gate 2 \\
\hline $0 \mathrm{~d}$ & 0 & 0 & 0 & 0 & gate 2 \\
\hline $0 \mathrm{e}$ & 0 & 1 & 0 & 2 & gate 2 \\
\hline $0 \mathrm{f}$ & 0 & 0 & 2 & 2 & gate 1 \\
\hline $0 \mathrm{~g}$ & 0 & 0 & 0 & 2 & gate 1 \\
\hline $0 \mathrm{~h}$ & 0 & 0 & 0 & 0 & gate 1 \\
\hline $0 \mathrm{i}$ & 0 & 1 & 0 & 0 & gate 2 \\
\hline $0 \mathrm{j}$ & 0 & 0 & 0 & 1 & gate 1 \\
\hline $1 \mathrm{a}$ & 0 & 0 & 0 & 1 & gate 2 \\
\hline $1 \mathrm{~b}$ & 0 & 1 & 0 & 1 & gate 2 \\
\hline $1 \mathrm{c}$ & 0 & 1 & 2 & 2 & gate 1 \\
\hline $1 \mathrm{~d}$ & 0 & 1 & 0 & 2 & gate 1 \\
\hline $1 \mathrm{e}$ & 0 & 1 & 0 & 0 & gate 1 \\
\hline $1 \mathrm{f}$ & 0 & 1 & 0 & 1 & gate 1 \\
\hline
\end{tabular}


Though the 2-parity 2 gate problem is trivial in comparison with the 12-parity 100 gate problem analyzed in [63] and [64], the graphs in Fig. 21(a) and (b) do show some of the findings in [64]. These include the fact that the solutions are unevenly distributed in the function space and the distribution is dependent on function lengths. Fig. 21(a) and (b) also show that the CGP representation is different from that used in [64]; as a result random sampling, or controlled random walks are likely to perform differently on using either representation.

\section{Modified OneMax and Deceptive Trap}

The coding for the modified versions of OneMax and deceptive trap function is identical to the usual version of the OneMax and deceptive trap function (explained in section A of this appendix), except for having an extra bit that indicates whether a genome is on the normal network, or on a parallel network of equal fitness.

The modified versions of OneMax and deceptive trap function shown in Fig. 20 (c), (d) and (e), are 4bits wide plus the extra indicator bit. The optimal fitness and the fitness of the neutral layers are 4 and 3 respectively for both functions. All strings of the form " $0 * * * *$ " (where * is a don't care term are on neutral layer. Strings starting with "1" are on the normal layer and have their fitness assigned the normal way.

\section{E. Hierarchical If-and-only-if Function}

For an n-bit binary string $x_{1} x_{2} \ldots x_{n}$, the hierarchical if-andonly-if (H-IFF) function is defined as [89], [90], [67]:

$$
\begin{aligned}
& f\left(x_{1}, x_{2}, \ldots, x_{n}\right)= \\
& \begin{cases}1 & \text { if } n=1 \\
n+f\left(x_{1}, \ldots, x_{n / 2}\right) & \text { if } n>1 \text { and }\left(\forall i: x_{i}=0\right. \\
\quad+f\left(x_{n / 2+1}, \ldots, x_{n}\right) & \text { or } \left.\forall i: x_{i}=1\right) \\
f\left(x_{1}, \ldots, x_{n / 2}\right) & \text { otherwise } \\
+f\left(x_{n / 2+1}, \ldots, x_{n}\right) & \end{cases}
\end{aligned}
$$

This function recursively decomposes a string into left and right halves. Each resultant sub-string gives a fitness contribution equal to its size if all the bits in the block have the same value (i.e. either all ones or all zeros). The fitness of the whole string is the sum of the fitness contributions for all substrings at all levels. Table 9 shows an example of how fitness is assigned for an 8-bit H-IFF. Fig. 23 is the quotient graph for the 8-bit H-IFF. Table 10 shows examples of genomes that belong to the search neutral sets of the 8 bit H-IFF of Fig. 23. Local optima are the 14 members of the quotient sets [16], [20] and [24]. The global optima are the all zero and all one strings, which are the members of set [32].

Table 9: Example fitness assignment for 8-bit H-IFF

\begin{tabular}{|l|l|l|l|l|l|l|l|r|}
\hline 1 & 1 & 1 & 1 & 1 & 0 & 1 & 1 & $8 * 1=8$ \\
\hline 1 & 1 & 1 & 1 & 1 & 0 & 1 & 1 & $3 * 2=6$ \\
\hline 1 & 1 & 1 & 1 & 1 & 0 & 1 & 1 & $1 * 4=4$ \\
\hline 1 & 1 & 1 & 1 & 1 & 0 & 1 & 1 & $0 * 8=0$ \\
\hline & \multicolumn{10}{|c|}{} & & & Total 18 \\
\hline
\end{tabular}

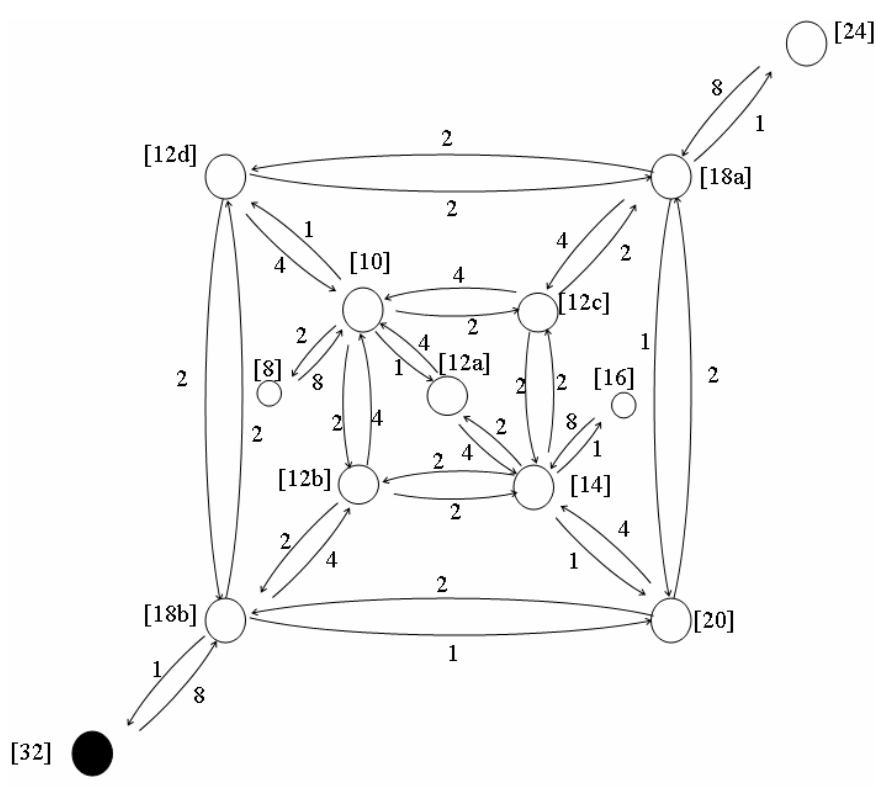

Fig. 23: Quotient graph for 8-bit HIFF

Table 10: Examples of genomes that belong to the search neutral sets of Fig. 23

\begin{tabular}{|l|llllllll|}
\hline Label & \multicolumn{7}{|l|}{ Genotype } \\
\hline 8 & 0 & 1 & 0 & 1 & 0 & 1 & 0 & 1 \\
\hline 10 & 0 & 0 & 0 & 1 & 0 & 1 & 0 & 1 \\
\hline $12 \mathrm{a}$ & 0 & 0 & 1 & 1 & 0 & 1 & 0 & 1 \\
\hline $12 \mathrm{c}$ & 0 & 0 & 0 & 1 & 0 & 1 & 1 & 1 \\
\hline $12 \mathrm{~b}$ & 0 & 0 & 0 & 1 & 0 & 0 & 0 & 1 \\
\hline 14 & 0 & 0 & 0 & 1 & 0 & 0 & 1 & 1 \\
\hline $12 \mathrm{~d}$ & 0 & 0 & 0 & 0 & 0 & 1 & 0 & 1 \\
\hline 16 & 0 & 0 & 1 & 1 & 0 & 0 & 1 & 1 \\
\hline $18 \mathrm{a}$ & 0 & 0 & 0 & 0 & 0 & 1 & 1 & 1 \\
\hline $18 \mathrm{~b}$ & 0 & 0 & 0 & 0 & 0 & 0 & 0 & 1 \\
\hline 20 & 0 & 0 & 0 & 0 & 0 & 0 & 1 & 1 \\
\hline 24 & 0 & 0 & 0 & 0 & 1 & 1 & 1 & 1 \\
\hline 32 & 0 & 0 & 0 & 0 & 0 & 0 & 0 & 0 \\
\hline
\end{tabular}

\section{ACKNOWLEDGMENT}

The authors of this paper would like to express their thanks to the anonymous reviewers for all of their helpful comments that has improved the quality and scope of this paper.

\section{REFERENCES}

[1] M. O'Neill, Automatic Programming in an Arbitrary Language: Evolving Programs with Grammatical Evolution. $\mathrm{PhD}$ thesis, University of Limerick. 2001.

[2] C. Ryan, J.J. Collins and M. O'Neill, "Grammatical Evolution: Evolving Programs for an Arbitrary Language," Lecture Notes in Computer Science: Proceedings of the First European Workshop on Genetic Programming, pp. 83-95, 1998. 
[3] D. Wilson, Grammatical Evolution Based Data Mining for Network Intrusion Detection. PhD Dissertation, University of Toledo. April 2008.

[4] C. Ryan, M. O'Neill and J.J Collins, "Grammatical Evolution: Solving Trigonometric Identities," Proceedings of Mendel 1998: 4th International Mendel Conference on Genetic Algorithms, Optimization Problems, Fuzzy Logic, Neutral Networks, Rough Sets, pp. 111-119, 1998.

[5] C. Ryan, and M. O'Neill, "Grammatical Evolution: A Steady State Approach," Proceedings of the Second International Workshop on Frontiers in Evolutionary Algorithms, pp. 419-423, 1998.

[6] C. Ryan, and M. O'Neill, "Grammatical Evolution," IEEE Transactions On Evolutionary Computation, Vol. 5, No. 4, August 2001.

[7] M. O'Neill and C. Ryan, Grammatical Evolution: Evolutionary Automatic Programming in an Arbitrary Language, Kluwer Academic Publishers, 2003.

[8] J. O'Sullivan and C. Ryan, "An Investigation into the Use of Different Search Strategies with Grammatical Evolution," J.A. Foster et al. (Eds.): EuroGP 2002, LNCS 2278, pp. 268-277. Springer-Verlag Berlin Heidelberg 2002.

[9] D. Wilson and D. Kaur , "Knowledge Extraction from KDD'99 Intrusion Data Using Grammatical Evolution" WSEAS Transactions on Information Science and Applications. Issue 2 Vol. 4 pp. 237 -244. February 2007.

[10] D. Wilson and D. Kaur, "Fuzzy Classification using Grammatical Evolution for Structure Identification", Proceedings of NAFIPS 2006, Montreal, June 3-6, 2006.

[11] D. Wilson, D. Kaur, M. Forrest and F. Lu, "A Grammatical Evolution Approach to System Identification of Laser Lap Welding', Proceedings of the SAE World Congress on Reliability and Robust Design in Automotive Engineering, pp. 493-497, Detroit, Aug 3-5, 2006.

[12] O'Neill, M., Cleary, R., Nikolov, N. 2004. Solving Knapsack Problems with Attribute Grammars Proceedings of the Third Grammatical Evolution Workshop GEWS 2004 ISGEC O'Neill M., Ryan C. Seattle, WA, USA.

[13] Cleary R., O'Neill M. 2005. An Attribute Grammar Decode for the 0/1 Multiconstrained Knapsack Problem European Conference on Evolutionary Combinatorial Optimisation EvoCOP 2005 34-45 LNCS 3448 Springer Raidl G.R., Gottlieb J. Lausanne, Switzerland.

[14] Cleary R. 2005. Extending Grammatical Evolution with Attribute Grammars: An Application to Knapsack Problems, Masters Thesis, University of Limerick.

[15] M. Kimura, Neutral theory of molecular evolution. Cambridge University Press, 1983.

[16] W. Banzhaf, "Genotype-phenotype-mapping and neutral variation - A case study in genetic programming," In Y. D. et al., editor, Parallel Problem Solving from Nature III, vol. 866 of LNCS, pp. 322-332, Oct. 1994.

[17] W. Banzhaf and A. Leier, "Evolution On Neutral Networks In Genetic Programming" Genetic Programming - Theory and Applications III T. Yu, R. Riolo and B. Worzel (Eds.), Kluwer Academic, Boston, MA, pp. 207 221, 2006.

[18] E. Galvan-Lopez and R. Poli, "An Empirical Investigation of How and Why Neutrality Affects Evolutionary Search" GECCO'06, Seattle, Washington, USA.July 8-12, 2006,

[19] Ryan, C., Keijzer, M., and Nicolau, M., "On the Avoidance of Fruitless Wraps in Grammatical Evolution". GECCO 2003, pp: 1752-1763, 2003.

[20] A. Aho and J. Ullman. "The theory of languages",.Mathematical Systems Theory, 2(2):97-125, 1968.

[21] A. McGettrick, "The Definition of Programming Languages", Cambridge Computer Science Texts vol 1, Cambridge University Press. 1980.

[22] J. Cohoon, S. Hegde, W. Martin and D. Richards, "Floorplan design using distributed genetic algorithms", Computer-Aided Design, ICCAD88. Digest of Technical Papers., IEEE International Conference, pp. 452455,1988

[23] S Ronald, J Asenstorfer and M Vincent "Representational Redundancy in Evolutionary Algorithms" , Proceedings of the 1995 IEEE International Conference on Evolutionary Computing, Volume 2, pg 631-637. 1995.

[24] N. Radcliffe and P. Surry, "Fitness Variance of Formae and Performance Prediction". FOGA3, pp.51-72. 1994.

[25] J Miller and P. Thomson, "Cartesian Genetic Programming" Proceedings of the 3rdEuropean Conferenceon Genetic Programming. LNCS Vol. 1802 pp. 121-132. 2000.
[26] J. Miller and S. Smith, "Redundancy and Computational Efficiency in Cartesian Genetic Programming", IEEE Transactions on Evolutionary Computation, Vol. 10, No. 2, pp. 167-174, 2006.

[27] P. Nordin and W. Banzhaf, "Complexity compression and evolution," In Eshelman, L., editor, Genetic Algorithms: Proceedings of the Sixth International Conference (ICGA95), pages 310-317, Pittsburgh, PA, USA. Morgan Kaufmann. 1995.

[28]C. R. Stephens and J.M. Vargas, "Effective fitness as an alternative paradigm for evolutionary computation I: General formalism," Genetic Programming and Evolvable Machines, 1(4):363-378. 2000.

[29] W. Banzhaf and W. B. Langdon, "Some considerations on the reason for bloat," Genetic Programming and Evolvable Machines, 3(1):81-91, 2002.

[30] D. Cvetkovic, P. Rowlinson, and C. Simic, Eigenspaces of Graphs. Encyclopedia of Mathematics and its Applications, Vol. 66, Cambridge University Press, 1997.

[31] P.F. Stadler, and G. Tinhofer, "Equitable partitions, coherent algebras, and random walks: applications to the correlation structure of landscapes," MATCH 40 pp.215-261, 2000.

[32] C. Godsil, Algebraic Combinatorics, Chapman and Hall, New York, NY. 1993.

[33] M. Shpak, P. F. Stadler, G. P. Wagner, and J. Hermisson. "Aggregation of variables and system decomposition: application to fittness landscape analysis," Theory in Biosciences 123: 33-68, 2004.

[34] Vose, M. D. The Simple Genetic Algorithm: Foundations and Theory. MIT Press, Cambridge, MA. 1999.

[35] Rowe J, Vose M D, Wright. "State Aggregation and Population Dynamics in Linear Systems" Artificial Life Vol. 11, No. 4, pp. 473-492. Fall 2005.

[36] J. Rowe, D. Whitley, L. Barbulescu and J. P. Watson, "Properties of Gray and binary representations" Evol. Comput. 12 47-76, 2004.

[37] Y. Borenstein and R. Poli, "Structure and metaheuristics", Proceedings of the 8th annual conference on Genetic and evolutionary computation, Seattle, Washington, USA July 08-12, 2006.

[38] F. Harary, "The Automorphism Group of a Hypercube" Journal of Computer Science, 6(1):pp. 136-138, 2000.

[39] D. Goldberg. "Genetic Algorithms in Search and Optimization", Addison-Wesley, 1989.

[40] Fontana, W., Schuster, P. "Continuity in evolution: On the nature of transitions". Science 280, pp. 1431-1433, 1998.

[41] Huynen, M.A., "Exploring phenotype space through neutral evolution". Molecular Evolution vol. 43, pp. 165-169, 1996.

[42] I. Harvey and A. Thompson. Through the labyrinth evolution finds a way: A silicon ridge. In Proceedings of the First International Conference on Evolvable Systems: From Biology to Hardware (ICES), pages 406-422. Springer-Verlag, 1996.

[43] Julstrom, B. A., "Redundant genetic encodings may not be harmful",Banzhaf, W. et al. , editors, GECCO99-1, Morgan Kaufmann Publishers, San Francisco, CA, pp.791, 1999.

[44] W. Banzhaf. Genotype-phenotype-mapping and neutral variation - A case study in genetic programming. In Y. D. et al., editor, Parallel Problem Solving from Nature III, volume 866 of LNCS, pages 322-332, Jerusalem, 9-14 Oct. 1994. Springer-Verlag.

[45] R. E. Keller and W. Banzhaf. Genetic programming using genotypephenotype mapping from linear genomes into linear phenotypes. In J. R. Koza, D. E. Goldberg, D. B. Fogel, and R. L. Riolo, editors, Genetic Programming 1996: Proceedings of the First Annual Conference, pages 116-122, Stanford University, CA, USA, 28-31 July 1996. MIT Press.

[46] T. Yu and J. Miller. Neutrality and the evolvability of boolean function landscape. In Fourth European Conference on Genetic Programming, pages 204-211. Springer-Verlag, 2001.

[47] M. Shackleton, R. Shipman, and M. Ebner. An investigation of redundant genotype-phenotype mappings and their role in evolutionary search. In Proceedings of the 2000 Congress on Evolutionary Computation CEC00 La Jolla Marriott Hotel pp. 493-500, 2000.

[48] L. Davis, Adapting operator probabilities in genetic algorithms. In Schaffer, J. D. (Ed.), Proceedings of the Third International Conference on Genetic Algorithms (pp. 61-69). San Mateo, CA: Morgan Kaufmann. 1989.

[49] L. J. Eshelman, and J. D. Schaffer. Preventing premature convergence in genetic algorithms by preventing incest. In Belew, R. K., \& Booker, L. B. 
(Eds.), Proceedings of the Fourth International Conference on Genetic Algorithms (pp. 115-122). San Mateo, CA: Morgan Kaufmann. 1991.

[50] S. Ronald, J. Asenstorfer, and M. Vincent, Representational redundancy in evolutionary algorithms. In 1995 IEEE International Conference on Evolutionary Computation, Vol. 2 pp. 631-636. 1995 .Piscataway, NJ.

[51] T. Smith, P. Husbands, and M. O'Shea. Neutral networks and evolvability with complex genotype-phenotype mapping. Lecture Notes in Computer Science, 2159:272-282, 2001.

[52] T. Smith, P. Husbands, and M. O'Shea. Neutral networks in an evolutionary robotics search space. In Congress on Evolutionary Computation: CEC 2001, pages 136-145. IEEE Press, 2001.

[53] R. Thomason and T. Soule "Redundant genes and the evolution of robustness", Proceedings of the 8th annual conference on Genetic and evolutionary computation. Seattle, Washington, USA pp. 959 - 960, 2006.

[54] Wilke CO, Wang JL, Ofria C, Lenski RE, Adami C: "Evolution of digital organisms at high mutation rates leads to survival of the flattest". Nature vol. 412, pp. 331-333, 2001

[55] Eigen, M., McCaskill, J. \& Schuster, P. "The molecular quasi-species." Adv. Chem. Phys. 75, pp. $149-263,1989$.

[56] Poli, R., and Galvan E., "On the Effects of Bit-Wise Neutrality on Fitness Distance Correlation, Phenotypic Mutation Rates and Problem Hardness", FOGA 2007.

[57] M. Toussaint, "On the evolution of phenotypic exploration distributions. In: Cotta, C., De Jong, K., Poli, R., Rowe, J. (eds.) Foundations of Genetic Algorithms 7 (FOGA 2003), pp. 169-182. Morgan Kaufmann, San Francisco. 2003.

[58] M. Toussaint. Self-adaptive exploration in evolutionary search. Technical Report IRINI 2001

[59] W. Banzhaf, W. B. Langdon, "Some Considerations on the Reason for Bloat", Genetic Programming and Evolvable Machines, v.3 n.1, p.81-91, March 2002

[60] K.E. Bassler, C. Lee, and Y. Lee, "Evolution of Developmental Canalization in Networks of Competing Boolean Nodes". Physical Review Letters, vol. 93, 038101., 2004.

[61] E. Galván López and R. Poli. Some Steps Towards Understanding How Neutrality Affects Evolutionary Search, in T. P. Runarsson, H. Beyer, E. K. Burke, J. J. Merelo Guervos, L D. Whitley and X. Yao, editors, PPSN IX: Proceedings of the 9th International Conference on Parallel Problem Solving from Nature, volume 4193 of LNCS, pages 778 - 787, Reykjavik, Iceland, 9 - 13 September 2006. Springer.

[62] C. O. Wilke, J. L. Wang, C. Ofria, R. E. Lenski, and C. Adami, "Evolution of digital organisms at high mutation rate leads to survival of the flattest". Nature, 412, pp. 331-333, 2001

[63] T. Yu and J. F. Miller. Needles in haystacks are not hard to find with neutrality. In J. A. Foster, E. Lutton, J. Miller, C. Ryan, and A. G. B. Tettamanzi, editors, Genetic Programming, Proceedings of the 5th European Conference, EuroGP 2002, volume 2278 of LNCS, pages 13-25, Kinsale, Ireland, 3-5 Apr. 2002. Springer-Verlag.

[64] M. Collins. Finding needles in haystacks is harder with neutrality. In H.G. Beyer, U.-M. O'Reilly, D. V. Arnold, W. Banzhaf, C. Blum, E. W. Bonabeau, E. Cantu-Paz, D. Dasgupta, K. Deb, J. A. Foster, E. D. de Jong, H. Lipson, X. Llora, S. Mancoridis, M. Pelikan, G. R. Raidl, T. Soule, A. M. Tyrrell, J.-P. Watson, and E. Zitzler, editors, GECCO 2005: Proceedings of the 2005 conference on Genetic and evolutionary computation, volume 2, pages 1613-1618, Washington DC, USA, 25-29 June 2005.

[65] http://www.worldwidewilson/cgp August 20, 2008.

[66] R. Shipman, M. Shackleton, and I. Harvey. The use of neutral genotypephenotype mappings for improved evolutionary search. BT. Technology Journal, 18(4):103-111, October. ISSSN 2000.

[67] M. Ebner, M. Shackleton and R. Shipman, "How Neutral Networks Influence Evolvability". Complexity, 7(2), pp. 19-33, Wiley Periodicals, 2002.

[68] J. D. Knowles and R. A. Watson, On the utility of redundant encodings in mutation-based evolutionary search. In Merelo, J. J., Adamidis, P., Beyer, H.-G., Fernandez-Villacanas, J.- L., \& Schwefel, H.-P. (Eds.), Parallel Problem Solving from Nature, PPSN VII (pp. 88-98), Berlin: SpringerVerlag, 2002
[69] M. O'Neill and C. Ryan, "Genetic code degeneracy: Implications for grammatical evolution and beyond," In ECAL '99: Proc. Of the Fifth European Conference on Artificial Life, Lausanne, Switzerland. 1999.

[70] M. O'Neill, C. Ryan and M. Nicolau, "Grammar Defined Introns: An Investigation Into Grammars, Introns, and Bias in Grammatical Evolution," In Spector, L., Goodman, E.D., Wu, A., Langdon, W.B., Voigt, H.-M., Gen, M., Sen, S., Dorigo, M., Pezeshk, S., Garzon, M.H., and Burke, E., editors, GECCO-2001: Proceedings of the Genetic and Evolutionary Computation Conference, pp. 97 - 103, San Fransisco, Carlifornia, USA. 2001.

[71] M. Nicolau, "Automatic grammar complexity reduction in grammatical evolution," In Poli et al, editors, In Proceedings of the Grammatical Evolution Workshop, Seattle, Washington, USA. 26-30 June, 2004.

[72] F Rothlauf and M Oetzel: "On the Locality of Grammatical Evolution",. Proceedings of the 5th European Conference on Genetic Programming (EuroGP 2006). pp. 320-330. 2006.

[73] M. O'Neill, A. Brabazon, M. Nicolau, S. McGarry, and P. Keenan, (2004). $\pi$ Grammatical Evolution, Proceedings of the Genetic and Evolutionary Computation Conference (GECCO 2004), LNCS 3103, Vol. 2, pp. 617-629, 2004.

[74] M O'Neill, A Brabazon: "mGGA: The meta-Grammar Genetic Algorithm". Proceedings of the 5th European Conference on Genetic Programming (EuroGP 2005). LNCS 3447, pp. 311-320, 2005.

[75] J. H. Holland. Adaptation in Natural and Artificial Systems. University of Michigan Press (Ann Arbor), 1975.

[76] M.D. Vose, "Generalizing the notion of schema in genetic algorithms. ", Artificial Intelligence, 50 1991, 385- 396.

[77] N. J. Radcliffe. "Equivalence class analysis of genetic algorithms." Complex Systems, 5(2):183-205, 1991.

[78] N. J. Radcliffe. "Genetic Set Recombination And Its Application To Neural Network Topology Optimization". Neural Computing and Applications, 1(1):67-90, 1993.

[79] N. J. Radcliffe. "Forma analysis and random respectful recombination". In Proceedings of the Fourth International Conference on Genetic Algorithms, pages 222-229. Morgan Kaufmann (San Mateo), 1991.

[80] N. J. Radcliffe, "The Algebra of Genetic Algorithms", Annals of Maths and Artificial Intelligence. Vol. 10, pp. 339- 384, 1994.

[81] F. Rothlauf and D. E. Goldberg," Redundant Representations in Evolutionary Computation", Evolutionary Computation, vol. 11, no. 4, pp. $381-415,2003$

[82] S. Choi and B. Moon. Normalization for Genetic Algorithms With Nonsynonymously Redundant Encodings. IEEE transactions on Evolutionary Computation.

[83] C. Ryan, M. Nicolau, and M. O'Neill, "Genetic algorithms using grammatical evolution," in Proc. 4th Eur. Conf. Genetic Program., EuroGP, 2002, LNCS 2278, pp. 279-288. 2002.

[84] A. Ortega, M. de la Cruz, and M. Alfonseca, "Christiansen Grammar Evolution: Grammatical Evolution With Semantics," IEEE Trans. On Evolutionary Computation, Vol. 11, No. 1, February 2007.

[85] Rothlauf, F: "Representations for Genetic and Evolutionary Algorithms". Studies in Soft Computing and Fuzziness, Volume 104.Heidelberg: Springer, June 2002.

[86] J. E. Rowe, "Population fixed-points for functions of unitation," in Foundations of Genetic Algorithms, W. Banzhaf and C. Reeves (eds.), vol. 5 pp. 69-84.Morgan Kaufmann: San Francisco, 1999.

[87] J. Richter, A. Wright and J. Paxton. "Exploration of Population Fixed Points Versus Mutation Rates for Functions of Unitation", Workshop on Evolutionary Computation Theory, GECCO-2004 June 26-30, 2004.

[88] T. Yu and J. Miller, "Through the Interaction of Neutral and Adaptive Mutations, Evolutionary Search Finds a Way". Artificial Life vol. 12, no. 4, pp. 525-551, 2006.

[89] R. A. Watson, G. S. Hornby, J. B. Pollack, "Modeling building-block interdependency". Parallel Problem Solving from Nature V; SpringerVerlag: Berlin, pp. 97-106, 1998.

[90] R. A. Watson, and J. B. Pollack, "Hierarchically-Consistent Test Problems for Genetic Algorithms", Procs. of 1999 CEC. Angeline, et al. eds. IEEE Press, pp.1406-1413, 1999. 